\title{
A Meta-Analysis of the Effect of Bacillus Calmette-Guérin Vaccination Against Bovine Tuberculosis: Is Perfect the Enemy of Good?
}

\section{OPEN ACCESS}

Edited by:

Federico Blanco,

National Institute of Agricultural

Technology (INTA), Argentina

Reviewed by:

Ramon A. Juste,

NEIKER Basque Institute for

Agricultural Research and

Development, Spain

Rebecca Lee Smith,

University of Illinois at

Urbana-Champaign, United States

*Correspondence:

Vivek Kapur

vkapur@psu.edu

Specialty section:

This article was submitted to Veterinary Infectious Diseases,

a section of the journal

Frontiers in Veterinary Science

Received: 11 December 2020

Accepted: 27 January 2021

Published: 18 February 2021

Citation:

Srinivasan S, Conlan AJK, Easterling LA, Herrera C, Dandapat $P$, Veerasami M, Ameni G, Jindal N, Raj GD, Wood J, Juleff N, Bakker D, Vordermeier M and Kapur V (2021) A Meta-Analysis of the Effect of Bacillus Calmette-Guérin Vaccination Against Bovine Tuberculosis: Is Perfect the Enemy of Good?

Front. Vet. Sci. 8:637580. doi: 10.3389/fvets.2021.637580

\begin{abstract}
Sreenidhi Srinivasan ${ }^{1,2}$, Andrew J. K. Conlan ${ }^{3}$, Laurel A. Easterling ${ }^{4}$, Christian Herrera ${ }^{1,2}$, Premanshu Dandapat ${ }^{5}$, Maroudam Veerasami ${ }^{6}$, Gobena Ameni ${ }^{7}$, Naresh Jindal ${ }^{8}$, Gopal Dhinakar Raj ${ }^{9}$, James Wood ${ }^{3}$, Nick Juleff ${ }^{10}$, Douwe Bakker ${ }^{11}$, Martin Vordermeier ${ }^{12,13}$ and Vivek Kapur ${ }^{1,2 *}$

${ }^{1}$ Department of Animal Science, The Pennsylvania State University, University Park, PA, United States, ${ }^{2}$ The Huck Institutes of the Life Sciences, The Pennsylvania State University, University Park, PA, United States, ${ }^{3}$ Disease Dynamics Unit, Department of Veterinary Medicine, University of Cambridge, Cambridge, United Kingdom, ${ }^{4}$ School of Veterinary Medicine, University of Pennsylvania, Philadelphia, PA, United States, ${ }^{5}$ Indian Veterinary Research Institute, Eastern Regional Station, Kolkata, India, ${ }^{6}$ Cisgen Biotech Discoveries Pvt Ltd, Chennai, India, ${ }^{7}$ Aklilu Lemma Institute of Pathobiology, Addis Ababa University, Addis Ababa, Ethiopia, ${ }^{8}$ Department of Veterinary Public Health and Epidemiology, Lala Lajpat Rai University of Veterinary and Animal Sciences, Hisar, India, ${ }^{9}$ Translational Research Platform for Veterinary Biological, Tamil Nadu University of Veterinary and Animal Sciences, Chennai, India, ${ }^{10}$ The Bill \& Melinda Gates Foundation, Seattle, WA, United States,

${ }^{11}$ Technical Consultant and Independent Researcher, Lelystad, Netherlands, ${ }^{12}$ Animal and Plant Health Agency, Addlestone, United Kingdom, ${ }^{13}$ Centre for Bovine Tuberculosis, Institute for Biological, Environmental and Rural Sciences, University of Aberystwyth, Aberystwyth, United Kingdom
\end{abstract}

More than 50 million cattle are likely exposed to bovine tuberculosis (bTB) worldwide, highlighting an urgent need for bTB control strategies in low- and middle-income countries (LMICs) and other regions where the disease remains endemic and test-andslaughter approaches are unfeasible. While Bacillus Calmette-Guérin (BCG) was first developed as a vaccine for use in cattle even before its widespread use in humans, its efficacy against bTB remains poorly understood. To address this important knowledge gap, we conducted a systematic review and meta-analysis to determine the direct efficacy of BCG against bTB challenge in cattle, and performed scenario analyses with transmission dynamic models incorporating direct and indirect vaccinal effects ("herdimmunity") to assess potential impact on herd level disease control. The analysis shows a relative risk of infection of $0.75(95 \% \mathrm{Cl}$ : $0.68,0.82)$ in 1,902 vaccinates as compared with 1,667 controls, corresponding to a direct vaccine efficacy of 25\% (95\% Cl: 18 , 32). Importantly, scenario analyses considering both direct and indirect effects suggest that disease prevalence could be driven down close to Officially TB-Free (OTF) status $(<0.1 \%)$, if BCG were introduced in the next 10 -year time period in low to moderate $(<15 \%)$ prevalence settings, and that $50-95 \%$ of cumulative cases may be averted over the next 50 years even in high (20-40\%) disease burden settings with immediate implementation of BCG vaccination. Taken together, the analyses suggest that BCG vaccination may help accelerate control of bTB in endemic settings, particularly with early implementation in the face of dairy intensification in regions that currently lack effective bTB control programs.

Keywords: BCG vaccine, bovine tuberculosis, efficacy, cattle, control program 


\section{INTRODUCTION}

Bovine tuberculosis (bTB) is a chronic infectious disease of cattle that is predominantly caused by Mycobacterium bovis, a zoonotic agent (1). The disease remains endemic in most low- and middleincome countries (LMICs) where it negatively impacts livestock productivity and represents a significant threat to public health. A live attenuated strain of $M$. bovis, the "Bacille de Calmette et Guérin" (BCG), has been used for experimental vaccination of cattle against bTB since 1913, well before its first trials in humans (2). Following Calmette and Guérin's promising early reports demonstrating safety of BCG and BCG-induced protection of cattle against experimental challenge with $M$. bovis, several trials were carried out in different countries in the early 20th century to better define its efficacy (3). Despite the repeated demonstration of BCG vaccine-induced protection in cattle, field use was not pursued because of the incomplete protection reported and, more importantly, because as a live attenuated vaccine, BCG sensitizes animals to the current World Organisation for Animal Health (OIE)-recommended purified protein derivatives (PPD)based skin tests. This compromises the specificity of the standard tuberculin skin test and results in an inability to differentiate infected from vaccinated animals (DIVA) (4). Therefore, bTB control programs that use the OIE-prescribed tuberculin skin test also prohibit the use of BCG vaccination (5).

In the last decade, research in the field has focused on identifying antigens that are present in M. bovis and absent or not immunogenic in BCG. In particular, the antigens ESAT-6, CFP10 and $\mathrm{Rv} 3615 \mathrm{c}$ have shown promise for differential diagnosis of bTB $(4,6,7)$. The DIVA capability of these antigens has been demonstrated in both experimental and naturally infected animals, hence enabling the use of BCG vaccination as part of future bTB control programs $(8,9)$.

In order to assess the potential utility of BCG vaccination as a component of future bTB control programs, accurate estimates of its efficacy are first required. We sought to address this major knowledge gap through a systematic review and meta-analysis of the existing literature on efficacy of BCG vaccination against bTB in cattle. Vaccines can protect populations through two main modes of action-reducing the susceptibility of vaccinates to infection or by reducing the potential for transmission by vaccinates after infection. The latter effect is particularly relevant for BCG vaccination where reduction in pathology has been reported more frequently $(10,11)$ than sterilizing immunity. However, also estimating reduction in infectiousness requires either large scale field trials or carefully designed natural transmission studies (11). The vast majority of published efficacy studies of BCG in cattle have used experimental challenge models with a relatively high infectious dose that can only measure a reduction in susceptibility rather than assess the impact on transmission.

For our quantitative meta-analysis, we therefore focus on the effect of the vaccine to reduce susceptibility to infection (vaccine efficacy, $\varepsilon_{s}$ ) defined by the presence or absence of visible lesions and/or confirmed by culture. We also review the evidence that supports what the possible range of efficacy BCG may offer in terms of a reduction in infectiousness $\left(\varepsilon_{\mathrm{I}}\right)$ and explore the implications for disease control using a conceptual dynamic transmission model.

\section{RESULTS}

\section{Characteristics of Included Studies}

A total of 1,392 articles were screened, and 24 articles were included in the analyses (Figure 1) (12-35). In the instance that an article evaluated different doses and strains of BCG, different routes of vaccine administration, breeds of cattle, etc., each was considered as separate strata level data and included as a unique study. For instance, the study by Wedlock et al. (20) was extracted into three strata level data, representing the three different strains or variants of BCG tested (BCG Danish 1331, BCG Danish 1331 freeze-dried and BCG Pasteur 1173P2) (20). In total, 49 strata level data were extracted from the 24 publications included in the systematic review. The included publications spanned the time period from 1972 to 2018, and represented a total of 1,902 vaccinates compared against 1,667 control animals. All included studies are summarized in Table 1.

\section{Meta-Analysis}

A funnel plot of the log risk ratio against standard error was constructed to assess potential publication bias (Figure 2). This revealed a large degree of asymmetry suggesting the presence of publication bias. There was no obvious difference in symmetry between random-effects (RE) and fixed-effects (FE) funnel plots. Similarly, visual inspection of the predicted vs. empirical observations (Normal Q-Q plot) also did not show any major differences in data fit for RE and FE models (Supplementary Figure 1).

Given the suggestion of publication bias in the data, we focus on the RE model, which adjusts for variability between individual studies, as the more appropriate model to assess the relative risk

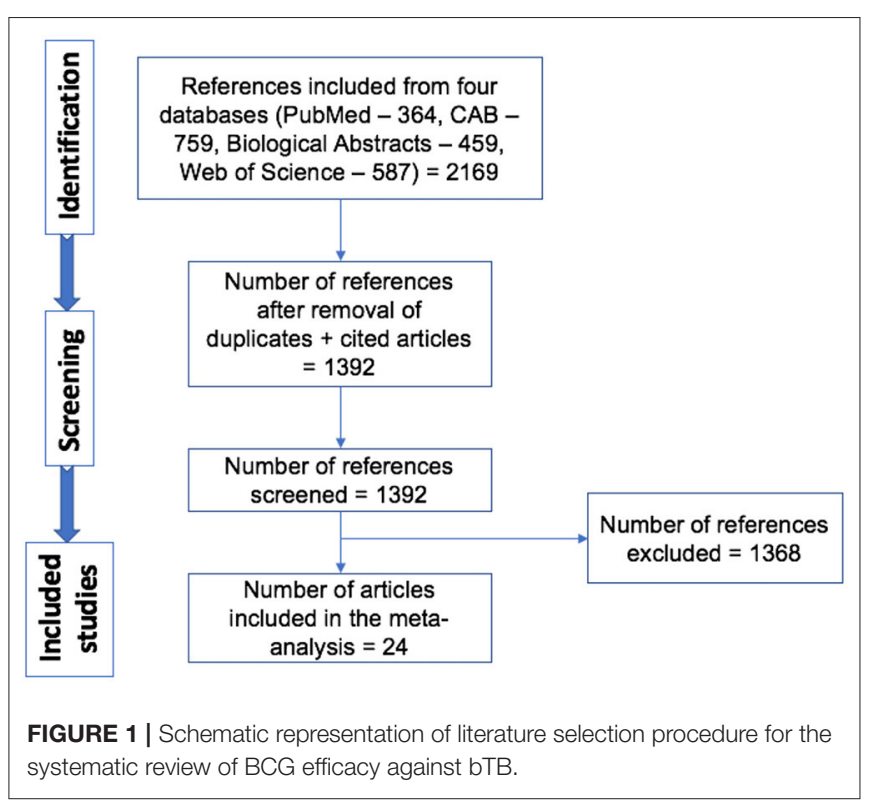


TABLE 1 | List of strata-level data $(n=49)$ extracted from a total of 24 publications for inclusion in our meta-analysis.

\begin{tabular}{|c|c|c|c|c|c|c|c|c|c|c|c|c|}
\hline $\begin{array}{l}\text { Database } \\
\#\end{array}$ & Authors & Source & Location & Tpos & Tneg & Cpos & Cneg & BCG dose & Route & Method & $\begin{array}{l}\text { Infection } \\
\text { measurement } \\
\text { method }\end{array}$ & $\begin{array}{l}\text { Sample allocation } \\
\text { method }\end{array}$ \\
\hline 101 & Wedlock et al. (20)_1 & BCG Pasteur 1173P2 & New Zealand & 9 & 1 & 10 & 0 & $1-4 \times 10^{6} \mathrm{CFU}$ & Subcutaneous & Experimental & Culture & Stratified Random Sample \\
\hline 101 & Wedlock et al. (20)_2 & BCG Danish 1331 & New Zealand & 8 & 2 & 10 & 0 & $1-4 \times 10^{6} \mathrm{CFU}$ & Subcutaneous & Experimental & Culture & Stratified Random Sample \\
\hline 101 & Wedlock et al. (20)_3 & $\begin{array}{l}\text { BCG Danish } \\
\text { freeze-dried }\end{array}$ & New Zealand & 9 & 1 & 10 & 0 & $1-4 \times 10^{6} \mathrm{CFU}$ & Subcutaneous & Experimental & Culture & Stratified Random Sample \\
\hline 120 & Buddle et al. (17) & BCG Pasteur 1173P2 & New Zealand & 16 & 2 & 7 & 2 & $1 \times 10^{5} \mathrm{CFU}$ & Subcutaneous & Experimental & Culture & Stratified Random Sample \\
\hline 579 & Ameni et al. (35) & BCG Danish 1331 & Ethiopia & 14 & 9 & 22 & 4 & $1-4 \times 10^{6} \mathrm{CFU}$ & Subcutaneous & Natural & Culture & Random Sample \\
\hline 802 & Buddle et al. (16) & BCG Pasteur 1173P2 & New Zealand & 5 & 4 & 5 & 4 & $5 \times 10^{5} \mathrm{CFU}$ & Subcutaneous & Experimental & Culture & Random Sample \\
\hline 826 & Buddle et al. (14)_1 & BCG Pasteur 1173P2 & New Zealand & 5 & 11 & 10 & 5 & $6 \times 10^{4} \mathrm{CFU}$ & Subcutaneous & Experimental & Culture & Not specified \\
\hline 826 & Buddle et al. (14)_2 & BCG Pasteur 1173P2 & New Zealand & 4 & 12 & 10 & 5 & $6 \times 10^{6} \mathrm{CFU}$ & Subcutaneous & Experimental & Culture & Not specified \\
\hline 828 & Buddle et al. (15)_1 & BCG Pasteur 1173P2 & New Zealand & 4 & 5 & 6 & 3 & $2 \times 10^{5} \mathrm{CFU}$ & Subcutaneous & Experimental & Culture & Random Sample \\
\hline 828 & Buddle et al. (15)_2 & BCG Pasteur 1173P2 & New Zealand & 4 & 5 & 6 & 3 & $2 \times 10^{3} \mathrm{CFU}$ & Subcutaneous & Experimental & Culture & Random Sample \\
\hline 828 & Buddle et al. (15)_3 & BCG Pasteur 1173P2 & New Zealand & 3 & 6 & 6 & 3 & $2 \times 10^{5} \mathrm{CFU}$ & Intratracheal & Experimental & Culture & Random Sample \\
\hline 956 & Berggren (13) & BCG Glaxo & Malawi & 75 & 129 & 82 & 128 & $8-26 \times 10^{6} \mathrm{CFU}$ & Subcutaneous & Natural & Culture & Alternate calves vaccinated \\
\hline 1065 & Wedlock et al. (22) & BCG Pasteur 1173P2 & New Zealand & 5 & 5 & 7 & 3 & $1 \times 10^{6} \mathrm{CFU}$ & Subcutaneous & Experimental & PM & Stratified Random Sample \\
\hline 1080 & Buddle et al. (21)_1 & BCG Pasteur 1173P2 & New Zealand & 3 & 7 & 7 & 3 & $1 \times 10^{6} \mathrm{CFU}$ & Subcutaneous & Experimental & PM & Stratified Random Sample \\
\hline 1080 & Buddle et al. (21)_2 & BCG Pasteur 1173P2 & New Zealand & 2 & 8 & 7 & 3 & $1 \times 10^{9} \mathrm{CFU}$ & Oral & Experimental & PM & Stratified Random Sample \\
\hline 1080 & Buddle et al. (21)_3 & BCG Pasteur 1173P2 & New Zealand & 3 & 7 & 7 & 3 & * & $\begin{array}{l}\text { Subcutaneous } \\
\text { and oral }\end{array}$ & Experimental & PM & Stratified Random Sample \\
\hline 1213 & Buddle et al. (18)_1 & BCG Pasteur 1173P2 & New Zealand & 6 & 4 & 10 & 0 & $1 \times 10^{6} \mathrm{CFU}$ & Subcutaneous & Experimental & Culture & Random Sample \\
\hline 1213 & Buddle et al. (18)_2 & BCG Pasteur 1173P2 & New Zealand & 9 & 1 & 10 & 0 & $1 \times 10^{6} \mathrm{CFU}$ & Subcutaneous & Experimental & Culture & Random Sample \\
\hline 1213 & Buddle et al. (18)_3 & BCG Pasteur 1173P2 & New Zealand & 8 & 2 & 10 & 0 & $1 \times 10^{6} \mathrm{CFU}$ & Subcutaneous & Experimental & Culture & Random Sample \\
\hline 1263 & De Klerk et al. (24) & BCG Pasteur 1173P2 & South Africa & 8 & 6 & 9 & 4 & $3.2 \times 10^{7} \mathrm{CFU}$ & Intramuscular & Combined & Culture & Random Sample \\
\hline 1266 & Ameni et al. (23) & BCG Danish 1331 & Ethiopia & 4 & 9 & 11 & 3 & $1 \times 10^{6} \mathrm{CFU}$ & Subcutaneous & Natural & Culture & Random Sample \\
\hline 1296 & Thom et al. (28)_1 & BCG Danish 1331 & UK & 6 & 3 & 8 & 1 & $1-4 \times 10^{6} \mathrm{CFU}$ & Subcutaneous & Experimental & Culture & Stratified Random Sample \\
\hline 1296 & Thom et al. (28)_2 & BCG Danish 1331 & UK & 9 & 0 & 9 & 0 & $1-4 \times 10^{6} \mathrm{CFU}$ & Subcutaneous & Experimental & Culture & Stratified Random Sample \\
\hline 1303 & Parlane et al. (31)_1 & BCG Danish 1331 & New Zealand & 14 & 2 & 16 & 1 & $2-8 \times 10^{6} \mathrm{CFU}$ & Subcutaneous & Experimental & PM & Stratified Random Sample \\
\hline 1303 & Parlane et al. (31)_2 & BCG Danish 1331 & New Zealand & 11 & 4 & 16 & 1 & $2-8 \times 10^{6} \mathrm{CFU}$ & Subcutaneous & Experimental & PM & Stratified Random Sample \\
\hline 1304 & Buddle et al. (29)_1 & BCG Danish 1331 & New Zealand & 2 & 7 & 10 & 0 & $1-4 \times 10^{5} \mathrm{CFU}$ & Subcutaneous & Experimental & PM & Stratified Random Sample \\
\hline 1304 & Buddle et al. (29)_2 & BCG Danish 1331 & New Zealand & 3 & 6 & 10 & 0 & $1-4 \times 10^{6} \mathrm{CFU}$ & Subcutaneous & Experimental & PM & Stratified Random Sample \\
\hline 1337 & Buddle et al. (33) & BCG Danish 1331 & New Zealand & 6 & 6 & 7 & 5 & $1.5 \times 10^{6} \mathrm{CFU}$ & Subcutaneous & Experimental & PM & Stratified Random Sample \\
\hline 1358 & Nugent et al. (34)_1 & BCG Danish 1331 & New Zealand & 0 & 30 & 8 & 122 & $1 \times 10^{8} \mathrm{CFU}$ & Oral & Natural & PM & Random Sample \\
\hline 1358 & Nugent et al. (34)_2 & BCG Danish 1331 & New Zealand & 1 & 33 & 8 & 122 & $3 \times 10^{5} \mathrm{CFU}$ & Subcutaneous & Natural & PM & Random Sample \\
\hline 1358 & Nugent et al. (34)_3 & BCG Danish 1331 & New Zealand & 3 & 169 & 9 & 108 & $1 \times 10^{8} \mathrm{CFU}$ & Oral & Natural & PM & Random Sample \\
\hline 1358 & Nugent et al. (34)_4 & BCG Danish 1331 & New Zealand & 11 & 166 & 12 & 74 & $1 \times 10^{8} \mathrm{CFU}$ & Oral & Natural & PM & Random Sample \\
\hline 1358 & Nugent et al. (34)_5 & BCG Danish 1331 & New Zealand & 12 & 156 & 26 & 83 & $1 \times 10^{8} \mathrm{CFU}$ & Oral & Natural & PM & Random Sample \\
\hline
\end{tabular}




\begin{tabular}{|c|c|c|c|c|c|c|c|c|c|c|c|c|}
\hline $\begin{array}{l}\text { Database } \\
\#\end{array}$ & Authors & Source & Location & Tpos & Tneg & Cpos & Cneg & BCG dose & Route & Method & $\begin{array}{l}\text { Infection } \\
\text { measurement } \\
\text { method }\end{array}$ & $\begin{array}{l}\text { Sample allocation } \\
\text { method }\end{array}$ \\
\hline 1358 & Nugent et al. (34)_6 & BCG Danish 1331 & New Zealand & 4 & 93 & 8 & 81 & $1 \times 10^{8} \mathrm{CFU}$ & Oral & Natural & PM & Random Sample \\
\hline 1366 & Buddle et al. (19)_1 & BCG Pasteur 1173P2 & New Zealand & 6 & 4 & 9 & 1 & $1 \times 10^{8} \mathrm{CFU}$ & Oral & Experimental & PM & Stratified Random Sample \\
\hline 1366 & Buddle et al. (19)_2 & BCG Pasteur 1173P2 & New Zealand & 7 & 3 & 9 & 1 & $1 \times 10^{9} \mathrm{CFU}$ & Oral & Experimental & PM & Stratified Random Sample \\
\hline 1366 & Buddle et al. (19)_3 & BCG Pasteur 1173P2 & New Zealand & 5 & 5 & 9 & 1 & $1 \times 10^{6} \mathrm{CFU}$ & Subcutaneous & Experimental & PM & Stratified Random Sample \\
\hline 1371 & Hope et al. (27)_1 & BCG Danish 1331 & UK & 7 & 0 & 7 & 0 & $2 \times 10^{6} \mathrm{CFU}$ & Subcutaneous & Experimental & PM and Culture & Not specified \\
\hline 1371 & Hope et al. (27)_2 & BCG Pasteur 1173P2 & UK & 3 & 4 & 7 & 0 & $2 \times 10^{6} \mathrm{CFU}$ & Subcutaneous & Experimental & PM and Culture & Not specified \\
\hline 1373 & Lopez-Valencia et al. (25) & BCG Tokyo & Mexico & 6 & 59 & 15 & 51 & $1 \times 10^{6} \mathrm{CFU}$ & Subcutaneous & Natural & $\begin{array}{l}\text { Skin test and IFNg } \\
\text { assay }\end{array}$ & Alternate calves vaccinated \\
\hline 1379 & Buddle et al. (26)_1 & BCG Danish 1331 & New Zealand & 2 & 7 & 6 & 4 & $1 \times 10^{6} \mathrm{CFU}$ & Subcutaneous & Experimental & $\mathrm{PM}$ & Stratified Random Sample \\
\hline 1379 & Buddle et al. (26)_2 & BCG Danish 1331 & New Zealand & 5 & 4 & 6 & 4 & $1 \times 10^{8} \mathrm{CFU}$ & Oral & Experimental & PM & Stratified Random Sample \\
\hline 1379 & Buddle et al. (26)_3 & BCG Danish 1331 & New Zealand & 5 & 4 & 6 & 4 & $1 \times 10^{7} \mathrm{CFU}$ & Oral & Experimental & PM & Stratified Random Sample \\
\hline 1379 & Buddle et al. (26)_4 & BCG Danish 1331 & New Zealand & 6 & 3 & 6 & 4 & $1 \times 10^{6} \mathrm{CFU}$ & Oral & Experimental & PM & Stratified Random Sample \\
\hline 1383 & Dean et al. (30) & BCG Danish 1331 & UK & 4 & 6 & 9 & 1 & $1 \times 10^{6} \mathrm{CFU}$ & Subcutaneous & Experimental & Culture & Not specified \\
\hline 1385 & Dean et al. (32)_1 & BCG Danish 1331 & UK & 8 & 2 & 8 & 1 & $1 \times 10^{6} \mathrm{CFU}$ & Subcutaneous & Experimental & Culture & Random Sample \\
\hline 1385 & Dean et al. (32)_2 & BCG Danish 1331 & UK & 5 & 5 & 8 & 1 & $5 \times 10^{5} \mathrm{CFU}$ & $\begin{array}{l}\text { Subcutaneous } \\
\text { and endobronchial }\end{array}$ & Experimental & Culture & Random Sample \\
\hline 1385 & Dean et al. (32)_3 & BCG Danish 1331 & UK & 5 & 5 & 8 & 1 & $1 \times 10^{6} \mathrm{CFU}$ & Endobronchial & Experimental & Culture & Random Sample \\
\hline 1410 & Nugent et al. (36) & BCG Danish 1331 & New Zealand & 2 & 518 & 8 & 289 & $3 \times 10^{5} \mathrm{CFU}$ & Subcutaneous & Natural & PM and Culture & Block Randomization \\
\hline
\end{tabular}

*Buddle et al. (21)_3 used a combination of Oral and Subcutaneous BCG; Study no. 1263 (24) was performed in buffaloes. Tpos and Cpos are animals classified as positive for bTB in vaccinates and controls, respectively. Theg and Cneg are animals that remained negative for bTB in vaccinates and controls, respectively.

Multiple strata level data extracted from a single study are represented with numbers at the end in the Author column. For example, Database \#101, Wedlock et al had 3 strata level data represented as Wedlock et al._1, Wedlock et al._2 and Wedlock et al._3. Buddle_a is Reference (14) and Buddle_b is Reference (15). a1, a2, and b1, b2 are strata level data of those respective studies. 

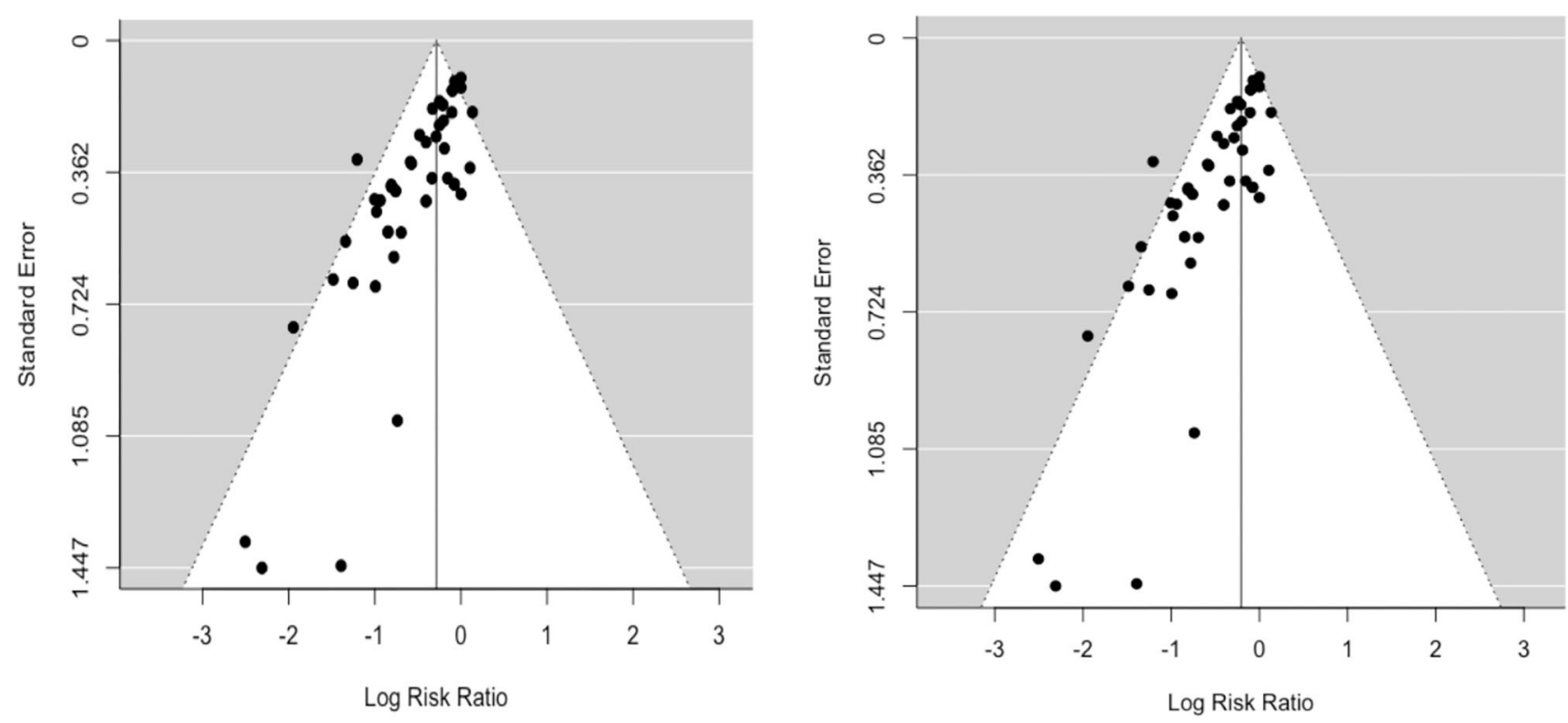

FIGURE 2 | Funnel plots (left: RE; right: FE) of the log risk ratio against standard error demonstrate potential publication bias.

ratio (RR). The relative risk ratio is defined as the probability of an outcome in an exposed group to that in an unexposed group (37). The RE model estimated RR to be 0.75 (95\% CI: 0.68 , 0.82 ), suggesting a $25 \%$ reduction in risk of infection (vaccine efficacy, $\varepsilon_{S}$ ) as measured by PM and/or culture in BCG vaccinates compared to control animals. Cochran's $(Q)$ value $(Q=76.1$, $d f=50$, and $p=0.01)$ and Higgins statistic $\left(I^{2}=32.1 \%\right)$ were computed to test for heterogeneity $\left(I^{2}<50 \%\right.$ represents low heterogeneity). While both the $Q$ value and $I^{2}$ statistic are classical measures of heterogeneity, neither is comprehensive since Cochran's $(Q)$ suffers low power and $I^{2}$ is imprecise in the case of meta-analyses of relatively small numbers of included studies. We used a forest plot to graphically summarize the variation in RR between studies (Figure 3).

To explore the potential impact of publication bias on the estimated RR, we carried out a sensitivity analysis using the trim and fill method (38). This is an algorithmic method to adjust for publication bias in a meta-analysis by imputing the values of missing studies (39). Here, the estimated number of missing studies on the right side of the funnel plot was found to be 21 (Figure 4). The Paul-Mantel method was used due to convergence issues with the maximum likelihood based methods (Maximum-Likelihood (ML), Restricted Maximum-Likelihood (40) and Empirical Bayes (EB) estimators). The adjusted RR estimate per this sensitivity analysis was found to be 0.84 (95\% CI: $0.73,0.98)$.

\section{Meta-Regression}

Several important biological factors varied between published studies, which a priori could have an impact on the estimated vaccine efficacy. Such confounding factors could also potentially lead to systematic patterns of bias as shown by the funnel plot analysis. We therefore constructed a multivariable metaregression model using these factors to explore whether they could improve model fit and to assess the relative effect of these biological variables on estimates of vaccine efficacy. The factors included BCG source, dose, route, whether or not revaccination was performed, and challenge method (Table 2).

An omnibus test of all the moderator variables ( $Q M=23.7$, $\mathrm{df}=13, p=0.03$ ) indicates that the explained variance by the model is greater than the unexplained variance. However, this amounts to only $49.1 \%$ of the total heterogeneity and only one moderator (BCG Glaxo source) has a RR significantly different than 1 (at the 95\% level). Given the relatively small sample sizes, reflective of the logistical constraints of experimental studies, this lack of statistical significance is unsurprising and does not rule out the potential biological importance of these variables and highlights the critical need for additional well-powered investigations to better assess the impact of these confounders on overall vaccine efficacy.

\section{Implications for bTB Control}

To explore the implications of the estimated efficacy of vaccination of $\varepsilon_{S} \sim 25 \%$ for disease control, we carried out scenario analyses using a conceptual herd level transmission dynamic model. Given the potential density dependence of transmission rates of bTB within herds, intensification of production in emerging dairy industries is a particular concern for LMICs (41). Thus, we consider a scenario with an initial herd size of 30 but growing in size at a rate of $15 \%$ per year to 134 animals after 50 years. Motivated by estimates from India we consider an initial bTB prevalence $(5,10$, and $15 \%)$ together with a set of higher prevalence scenarios (20,30, and 40\%) (42). There are currently no published quantitative estimates of transmission rates from LMICs, hence for this model population we use a 


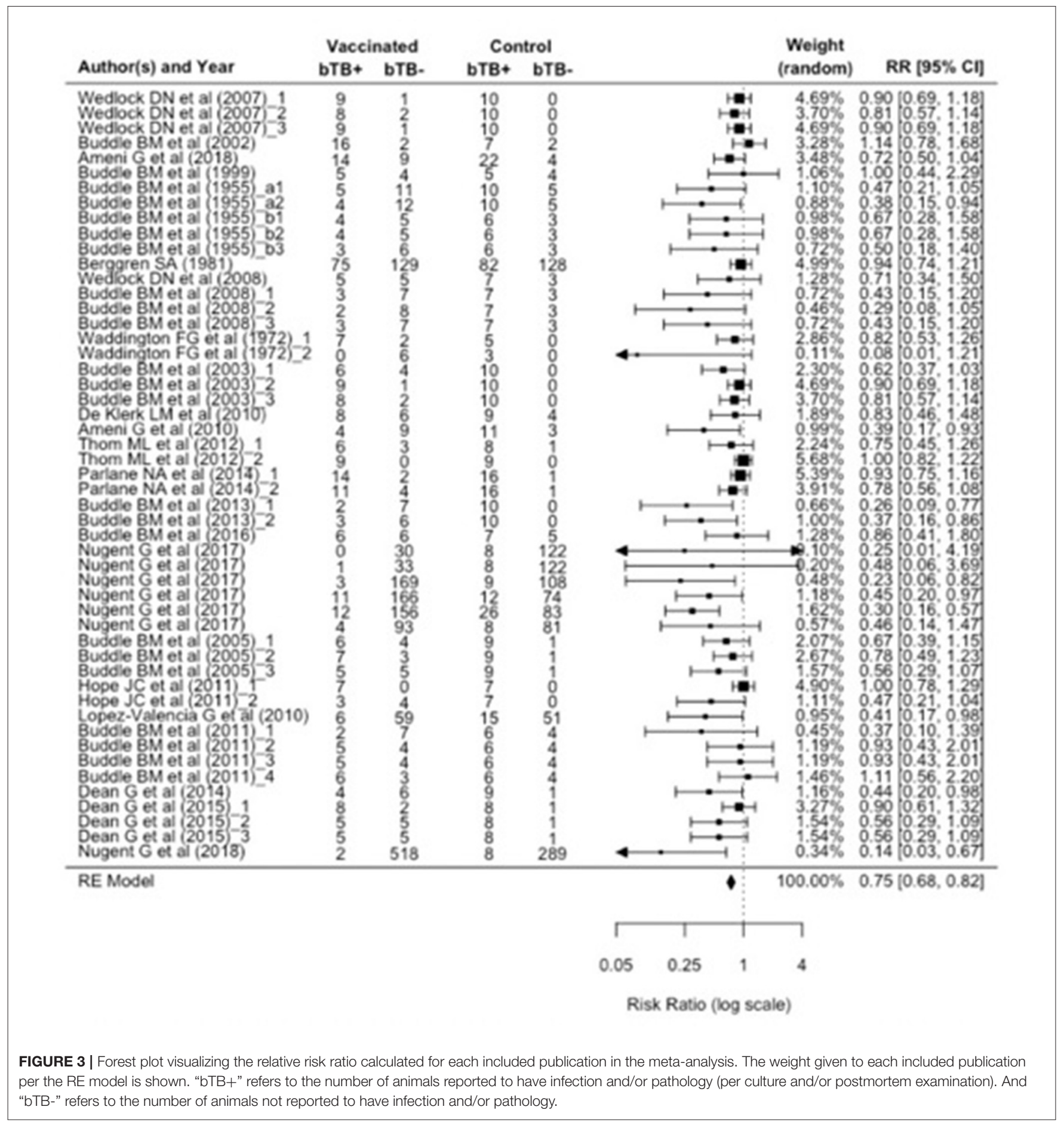

density dependent transmission function estimated from herds in Great Britain (11).

In the absence of estimates of indirect BCG effects based on empirical trials, we used data from long-term natural challenge models to estimate the relative contribution of indirect effects when force of infection is low (representative of field setting). Overall efficacy in those recent field trials included in this meta-analysis [excluding the older Berggren (13) study] was estimated to be of $61 \%$ (95\% CI: 40,74$)$ for natural transmission compared to $18 \%(95 \% \mathrm{CI}: 11,24)$ for experimental challenge studies (Table 3). The key distinction between experimental challenge and natural transmission studies-beyond being more representative of a field setting-is that the latter measures the total effect of vaccination (Figure 5). The total effect 


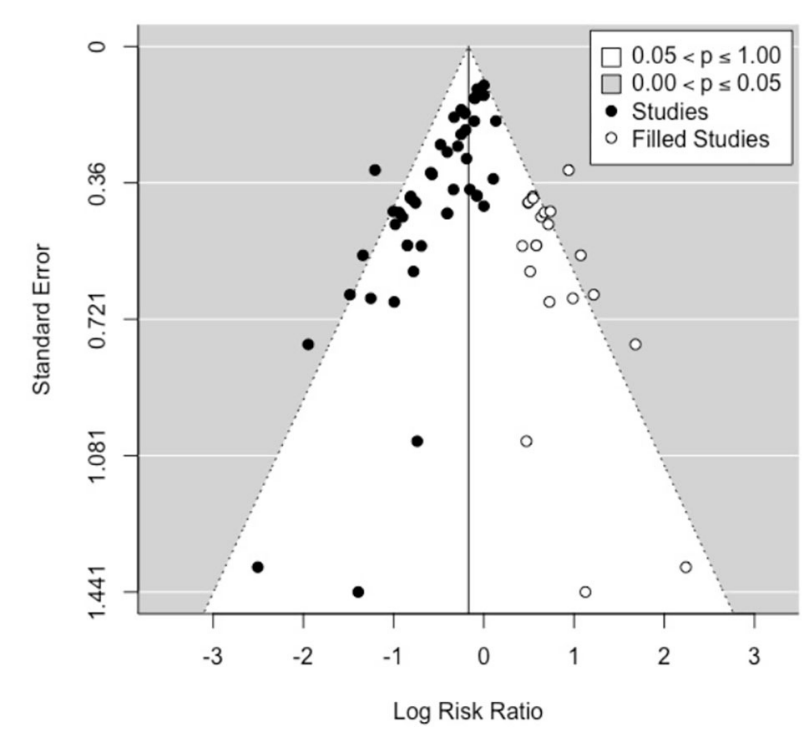

FIGURE 4 | Funnel plot showing the 21 studies (open circles, on the right side) imputed by the trim-and-fill method to carry out a sensitivity analysis for publication bias.

TABLE 2 | Multivariable meta-regression of the selected predictors on BCG efficacy $\left(\mathrm{R}^{2}=49.10 \%, n=49\right)$.

\begin{tabular}{|c|c|c|c|}
\hline Moderators & Categories & RR (95\% Cl) & $p$-value (RE) \\
\hline \multirow[t]{2}{*}{ Revaccination } & No & Reference & \\
\hline & Yes & $1.04(0.79,1.38)$ & 0.77 \\
\hline \multirow[t]{6}{*}{ BCG route } & Endobronchial & Reference & \\
\hline & Intramuscular & $2.16(0.55,8.50)$ & 0.27 \\
\hline & Intratracheal & $1.01(0.28,3.67)$ & 0.99 \\
\hline & Oral & $2.04(0.75,5.53)$ & 0.16 \\
\hline & Subcutaneous & $1.46(0.71,3.00)$ & 0.3 \\
\hline & $\begin{array}{l}\text { Subcutaneous and } \\
\text { Endobronchial }\end{array}$ & $1(0.38,2.66)$ & 1.00 \\
\hline \multirow[t]{2}{*}{ Challenge method } & Natural & Reference & \\
\hline & Experimental & $0.83(0.40,1.70)$ & 0.61 \\
\hline \multirow[t]{4}{*}{ BCG source } & Danish 1331 & Reference & \\
\hline & Danish freeze-dried & $1.14(0.77,1.68)$ & 0.51 \\
\hline & Glaxo & $12.14(1.52,97.09)$ & 0.02 \\
\hline & Pasteur 1173P2 & $0.98(0.77,1.23)$ & 0.84 \\
\hline BCG Dose & & $0.68(0.31,1.51)$ & 0.35 \\
\hline $\begin{array}{l}\text { Time from vaccination } \\
\text { to challenge }\end{array}$ & & $1.00(0.99,1.001)$ & 0.5 \\
\hline $\begin{array}{l}\text { Length of exposure to } \\
\text { challenge }\end{array}$ & & $0.998(0.997,1)$ & 0.06 \\
\hline
\end{tabular}

of vaccination will be greater than the direct protection of individuals $\left(\varepsilon_{s}\right)$ due to the reduction in transmission from herd immunity effects, along with any additional protection from reduction in infectiousness $\left(\varepsilon_{I}\right)$ of vaccinated individuals.

In the absence of control, the null model predicts a gradual increase in prevalence with herd size saturating around $~ 70 \%$ for all initial conditions (Figure 6). We considered a vaccine efficacy to reduce infectiousness $\left(\varepsilon_{\mathrm{I}}\right)$ at two hypothetical thresholds: (i) $\sim 36 \%$, approximating the difference between average overall efficacy in the natural transmission studies (0.61) and the overall direct efficacy as revealed by the meta-analyses of 0.25 ; and (ii) $\sim 49 \%$ - representing the difference between the upper bound of efficacy in the natural transmission studies (0.74) and overall direct efficacy as revealed by the meta-analyses of 0.25 .

Our model scenarios illustrate the benefits of early intervention. Even with only a modest efficacy $\left(\varepsilon_{\mathrm{s}}\right)$ of $25 \%$, a window of up to 25 years of slightly lower than the current disease prevalence levels may be achieved (Figure 6A), should BCG be implemented immediately in the low to moderate prevalence scenario $(5,10,15 \%)$. With an additional reduction in infectiousness $\left(\varepsilon_{\mathrm{I}}\right)$ of vaccinates $(49 \%)$, disease prevalence could be driven down close to Officially TB Free (OTF) status $(<0.1 \%$ per the European Union) if BCG were introduced in the next 10-year time period in low to moderate prevalence settings. Immediate implementation, but with a lower $\varepsilon_{\mathrm{I}}$ of $36 \%$, prevalence may start to rise again in 30-40-years, driven by increased herd size, suggesting that other strategies will also be required.

These scenarios predict considerable benefits in terms of reducing cumulative cases if BCG is implemented now. With an impact of vaccination on infectiousness, between $\sim 80$ and $100 \%$ of cases can be saved in 50 years, driven by the strength of indirect effects on transmission (Figure 6B). Encouragingly, this model also predicts that from $\sim 50$ to $95 \%$ of cumulative cases can be averted in higher disease burden settings $(>15 \%)$ if acted upon now (Figure 6).

\section{DISCUSSION}

Following promising initial studies conducted by Calmette, Guérin and others that showed both safety of BCG and BCG-induced protection of cattle against both natural and experimental challenge with $M$. bovis, several trials were carried out through the early and mid 20th century in many different countries to better assess and define the efficacy of ancestral variants of BCG in cattle and showed varying levels of protection $(3,44)$ (Supplementary Table 1). While BCG went on to become the most widely used of all human vaccines (and the only available vaccine against human TB), it has not been considered for routine use in domestic livestock despite early promise. This is primarily because of the fact that BCG sensitizes animals to the widely used and OIE-recommended PPD based skin tests, which precludes its use where skin test-based control is being actively pursued (5), and it is with this context that an Expert Committee of the WHO/FAO Expert Committee on Zoonoses (45) stated that: "generally speaking, vaccination has no place in the eradication of tuberculosis in cattle."

The early proof-of-concept experiments conducted by Calmette and Guérin in the early 1900s to demonstrate safety of BCG and protection against progressive TB in cattle were replicated and refined by several other investigators in both Europe and the Americas (44, 46-53). Their findings led to considerable international interest in exploring the possibility 
TABLE 3 | Natural transmission studies included in the meta-analysis.

\begin{tabular}{|c|c|c|c|c|c|c|c|c|}
\hline Database \# & Authors & Location & BCG source & BCG route & BCG dose & Controls_n & Vaccinates_n & $\begin{array}{c}\text { Reported } \\
\text { efficacy }\end{array}$ \\
\hline 579 & Ameni et al. (35) & Ethiopia & BCG Danish 1331 & Subcutaneous & $1-4 \times 10^{6} \mathrm{CFU}$ & 26 & 23 & $30 \%$ \\
\hline 1266 & Ameni et al. (23) & Ethiopia & BCG Danish 1331 & Subcutaneous & $1 \times 10^{6} \mathrm{CFU}$ & 14 & 13 & $60 \%$ \\
\hline 1358 & Nugent et al. (34) & New Zealand & BCG Danish 1331 & Oral and Subcutaneous & $1 \times 10^{8} \mathrm{CFU}$ & 531 & 644 & $67 \%$ \\
\hline 1373 & $\begin{array}{l}\text { Lopez-Valencia et al. } \\
\text { (25) }\end{array}$ & Mexico & BCG Tokyo & Subcutaneous & $1 \times 10^{6} \mathrm{CFU}$ & 66 & 65 & $60 \%$ \\
\hline 1410 & Nugent et al. (36) & New Zealand & BCG Danish 1331 & Subcutaneous & $3 \times 10^{5} \mathrm{CFU}$ & 297 & 520 & $85 \%$ \\
\hline
\end{tabular}

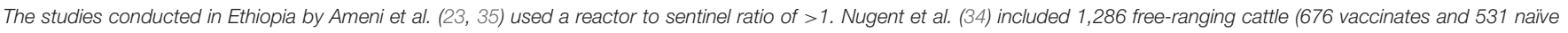

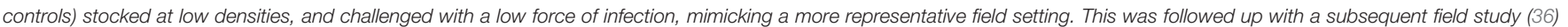

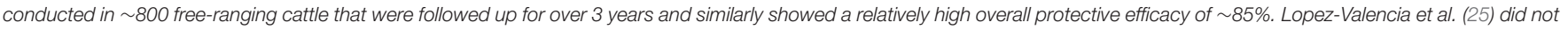
conduct necropsy.

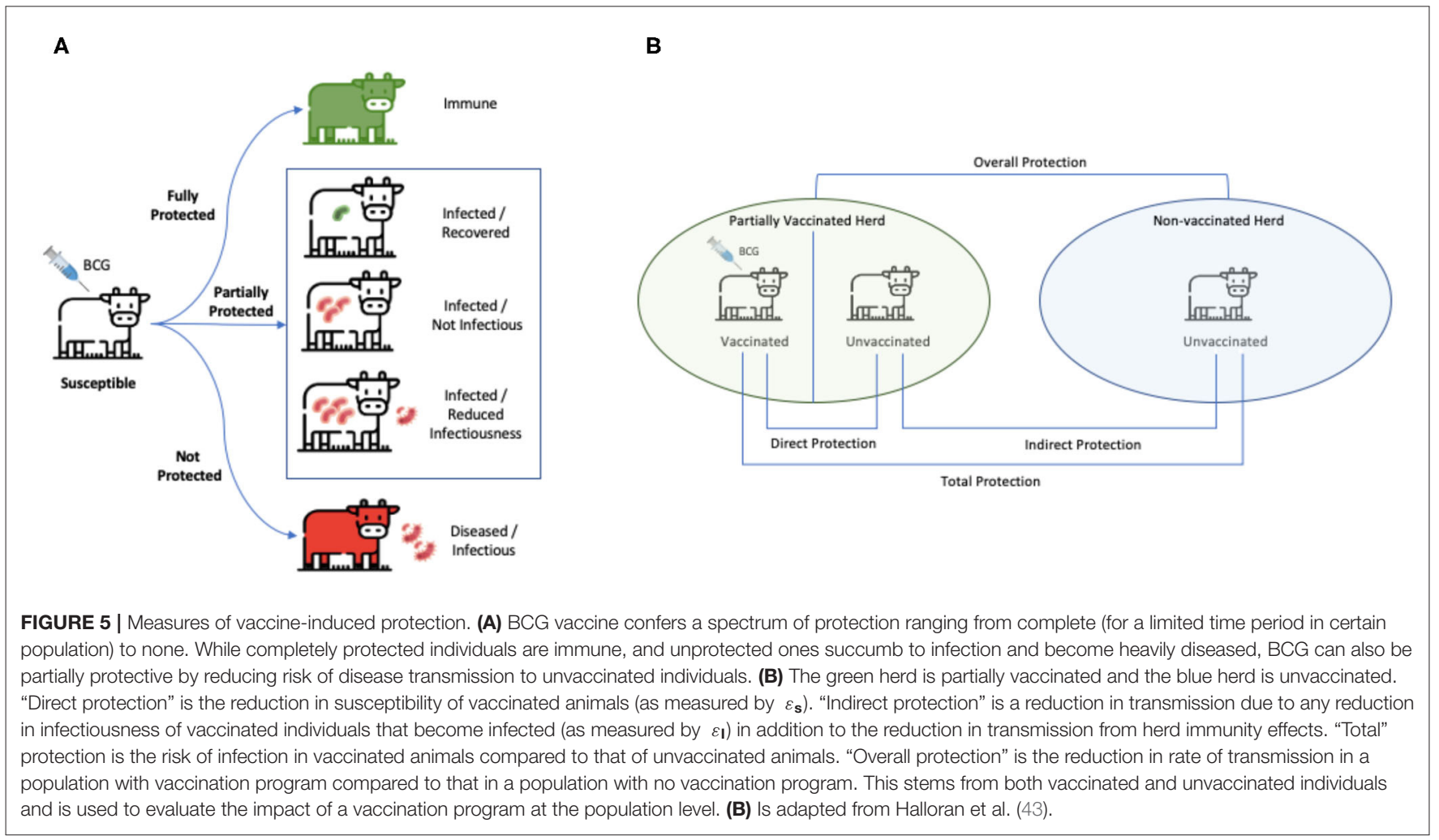

of eradicating bTB by vaccinating cattle with BCG as has been reviewed elsewhere $(3,10,54-57)$. As part of the current systematic review, we extracted data from eight studies that were published between 1930 and 1972 (Supplementary Table 1). However, given that these studies used ancestral strains of BCG and doses that are not possible to accurately estimate, these studies were excluded from the formal analyses. However, it is noteworthy that these studies provided key foundational evidence that highlighted both the variable protection afforded by BCG in experimental and natural challenge, as well as showed that BCG vaccination sensitizes animals to tuberculins and hence would confound ongoing test and slaughter tuberculosis eradication programs. However, recent attempts to develop BCG-compatible diagnostic tests have shown great promise and paved the way for implementation of BCG-based control programs in LMICs and other endemic regions (7-9). Thus, there is renewed interest in exploring the use of BCG vaccination as a component of national control programs, particularly where test and slaughter is unfeasible $(58,59)$.

We systematically reviewed the literature to obtain estimates of the efficacy of BCG against bTB and assessed its potential utility in future control programs. The analyses suggest an overall vaccine efficacy of $25 \%$ (95\% CI: 18 , 32) as measured by the presence of visible lesions and/or culture. The potential contribution of vaccine strain as a confounder or predictor of BCG efficacy is intriguing. This is relevant since a majority of the studies that were included in the current review used either BCG Pasteur 1173P2 


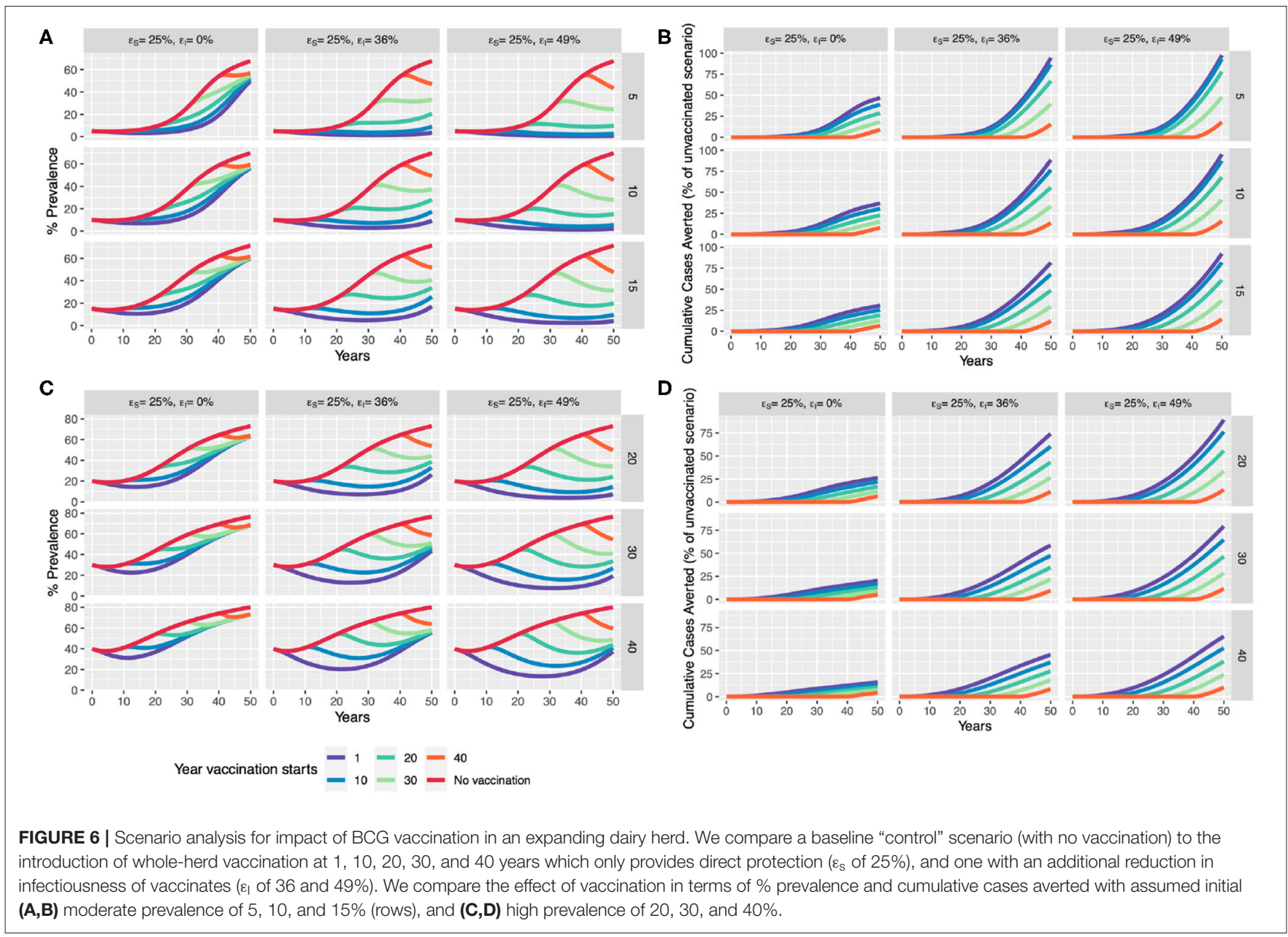

(9 out of 24 studies) or BCG Danish 1331 (10 out of 24 studies). The meta-regression model suggests that neither of these strains had any significant effect on the observed heterogeneity. However, given the small sample size, further investigations may be warranted to assess the robustness of these inferences.

Given the large variability in the age at which animals were vaccinated in the included studies, the ideal timing for vaccination could not be subjected to rigorous analysis. While there is evidence in the published literature showing that BCG vaccination within a few days of birth $(<1$ month of age) can induce high levels of protective immunity $(18,60)$, there is a need for more studies in relevant settings, such as in bTB-endemic regions where high burden of environmental mycobacteria has been reported (61). In these settings, some studies suggest prior sensitization with environmental mycobacteria prevent induction of protection in calves, while others report that exposure to $M$. avium helps induce protection against $M$. bovis $(17,62)$. It is plausible that these seemingly contradictory results could be because exposure to different environmental mycobacteria could either enhance or reduce BCG's efficacy, and hence, future studies are needed to help clarify the role of these confounders to vaccine efficacy. Curiously, despite the early recognition by Calmette and Guerin and other investigators of the need for revaccination (at annual or 18 month intervals) in order to maintain protection (3), only a total of five studies performed revaccination, highlighting the scarcity of reliable data available on duration of immunity and revaccination intervals to formulate evidence-based approaches to control bTB $(13,16$, $18,24,31)$. Thus, it is important to assess the true duration of immunity and role, if any, of revaccination to obtain better estimates of the efficacy of BCG and inform the parameterization of robust transmission dynamics and econometric models that may be used to guide development and implementation of future cost-effective vaccine-based intervention strategies $(18,31)$.

The roles of dose and type of challenge (experimental challenge or natural infection) were also investigated. A total of 17 studies that are part of this meta-analysis performed experimental challenge of their animals to infection. These studies call for high dose infection protocols in order to generate high levels of infection in control animals, which do not reflect the reality in natural conditions where animals most likely are not exposed to such high numbers of virulent bacteria as a single bolus. Such experimental challenge models with high infection doses do not allow BCG protection to be revealed. One reason for this may be that these models are often used 
for the development of novel vaccines aimed at improving BCG efficacy and not per se to study BCG efficacy. This highlights the importance of conducting robust natural transmission studies in field conditions and in endemic settings where the real need for vaccination lies. It is noteworthy that most experimental trials have used M. bovis as the challenge strain. However, $M$. bovis is not the only causative agent of bTB since $M$. tuberculosis and other members of the M. tuberculosis complex are frequently isolated from cattle samples, and certain species of non-tuberculous mycobacteria (NTM) that contain virulence factors encoded in the Region of Difference- 1 are also known to cause TB-like lesions $(63,64)$. Hence, future studies are needed to better estimate efficacy of BCG vaccine-induced protection with locally relevant bTB causing mycobacteria other than M. bovis.

It is important to note that there are several limitations to systematic reviews and meta-analyses. They can provide a summary of published evidence about a specific question, but our ability to answer it is limited by variation in experimental design and the consistency and completeness of reporting. This systematic review was limited to studies in English indexed in the four databases screened, and hence may have missed relevant investigations. Studies that did not report essential details were excluded. It is not surprising that systematic biases in the fit of the meta-analysis models suggest existence of publication bias and the potential to inflate or underestimate estimates of direct vaccine efficacy. One of the primary limitations of the included studies is that the reported measures of protection, evidence of lesions at necropsy and culture, together with immune reactions, are the current "gold standards," but have variable sensitivity and specificity (65). In order to address any potential bias in measurement of the outcome, we focused on studies which reported culture or necropsy data for calculation of RR estimation and further analyses. An exception was made however for the Lopez-Valencia et al. (25) study since it was one of only five estimates from a recent natural transmission study. This study considered an animal to be infected if there were positive reactions to the tuberculin skin test and the IFN $\gamma$ release assay upon stimulation with PPD-B and recombinant ESAT6-CFP10 (25). While many studies followed a simple or stratified sampling strategy for randomization, two studies $(13,25)$ used a more systematic approach of vaccinating every alternate calf in the herd which may have introduced potential biases from the randomization process (Table 1). However, a rigorous assessment of other risks of bias due to deviations from intended interventions, missing outcome data and selection of reported result could not be performed due to lack of access to the complete data or detailed experimental protocols from these studies. Another limitation is that only the direct effect of vaccination (reduction in susceptibility, $\varepsilon_{S}$ ) could be estimated from the experimental challenge experiments (66). This is important, in particular, given our scenario analyses which demonstrate the transformative effect an additional reduction in infectiousness of vaccinates $\left(\varepsilon_{I}\right)$ could have $(67,68)$. Thus, studies to assess the impact of BCG on infectiousness of vaccinates through natural transmission experiments or field trials are urgently needed (67).
Herd-size is perhaps the most important risk factor associated with transmission of bTB (69), leading to the common assumption that transmission is density dependent and increases with herd size $(70,71)$. This poses a particular challenge for bTB control in emerging dairy markets in LMICs where intensification of the dairy sector may make achieving control increasingly difficult over time. We developed herd level transmission dynamic models and performed scenario analyses using deterministic models to illustrate this challenge. The results of these analyses are striking - and suggest that despite the relatively modest direct protection afforded by BCG, a strong case may be made for implementing vaccination for bTB control sooner rather than later, with overall benefits reducing progressively over time to intervention (72) (Figure 6). Overall, a leaky vaccine like BCG could still play a pivotal role in disease control, particularly if implemented sooner than later in the face of dairy intensification efforts in bTB endemic countries.

Finally, even while scenario analyses are for illustrative purposes, there are several limitations to these analyses that we recognize and should be addressed in future investigations. To begin with, in the absence of surveillance programs in LMICs, transmission estimates are lacking and should be determined to accurately assess risk. Given that recent studies show increasing prevalence in intensifying cattle herds in endemic settings (69), these observations have major implications for informing and implementing disease control policy and suggest a potential role for BCG vaccination that has hitherto remained unexplored. Next, in order to realize herd-level benefits of vaccination, estimating the reduction in the risk of transmission from vaccinated individuals $\left(\varepsilon_{I}\right)$ is crucial. Animal trials in relevant endemic settings are urgently needed to evaluate the potential of BCG to reduce onward transmission in the field (11).

It is important to note that in addition to cattle, tuberculosis affects a wide variety of livestock and both free-living and captive wildlife species including goats, sheep, pigs, cervids, wild boars, badgers, brushtail possums, and ferrets, many of which are recognized as potential reservoir hosts. Indeed, experimental and field trials to study the response to BCG vaccination of some these species suggests that, while vaccination confers incomplete protection, it's use in domestic and captive or free-living wildlife species should be seriously considered to reduce risk of crossspecies spillover (73). Alternative approaches to control bTB, such as test-and-segregate and treatment with anti-mycobacterial agents including isoniazid have also been explored $(74,75)$. Treatment with isoniazid is uneconomical and not advised given the long duration of treatment needed and the need to withdraw milk together with reports that bacterial shedding may resume as soon as isoniazid is withdrawn (76). Moreover, use of a first line antimycobacterial agent in food animals is of major concern due to potential for contribution to the spread of drug resistant tuberculosis (77). Over the past decade, research in the field has also focused on improving $\mathrm{TB}$ vaccination using alternative approaches including the heterologous prime-boost strategy, introducing genetic modifications in BCG strains to increase immunogenicity, and also completely replacing BCG with attenuated $M$. bovis strains (78-82). Heat-inactivated $M$. bovis vaccines have also recently shown promise in wild boars, 
pigs, red deer, badgers, and goats (73, 83-88). However, despite significant efforts and promising results in preclinical studies, there is only limited evidence from clinical and field trials for significant gains in efficacy of these newer generation or modified BCG vaccines. Interestingly, both heat-inactivated and attenuated vaccines for another major mycobacterial disease, paratuberculosis (Johne's disease), have been licensed and tested extensively for use in cattle and small ruminants, but their use is limited in countries because of potential for interference with tuberculin testing and diagnostic tools currently used in bTB eradication and control programs $(89,90)$.

In conclusion, in the short-term, in endemic regions where test and slaughter approaches to bTB control have not been shown to be effective or, BCG vaccination alongside a DIVA diagnostic test appears to be the most promising option in the near future. However, it is important to continue to develop and assess next generation vaccines as well as complementary strategies of disease control alongside that of BCG. For instance, the assessment of routine testing and segregation of reactor animals, mandatory pasteurization of milk fed to calves and sold for human consumption, enhanced husbandry practices, such as segregation of reactor or likely infected animals, herd certification policies for recruitment of disease-free animals, slaughterhouse surveillance, regulating movement and trade of reactor animals, etc., in conjunction with or as alternatives to BCG need to be rigorously evaluated in different production settings and epidemiological contexts. Together, our studies highlight an urgent need as well to perform sensitivity analyses and build econometric frameworks to assess the cost-benefit impacts of implementing vaccine-based control strategies to establish the business case for (or against) implementation of BCG vaccination as a component of a national bovine TB control program.

\section{CONCLUSION}

This systematic review and meta-analysis of the efficacy of BCG vaccination in cattle together with transmission dynamic model-based scenario analyses provides strong evidence for the consideration of implementation of BCG vaccine-based bTB control strategies, particularly in LMICs and other high burden settings. Despite a relatively small but positive protective effect, conservative transmission models suggest an important role for BCG in limiting spread of the disease and buying time for improvement of vaccine efficacy or the development of alternative approaches to disease control. Taken together with the predicted increase in prevalence associated with intensification of dairy production, our investigations suggest that BCG vaccination may indeed be simply good (enough) to accelerate control of bTB in endemic settings.

\section{METHODS}

\section{Literature Search Strategy}

A systematic search was performed for published articles reporting the effect of BCG against bTB in cattle as of February 24, 2020. Various combinations of Boolean operators and $\mathrm{MeSH}$
TABLE 4 | Study inclusion/exclusion criteria.

\begin{tabular}{ll}
\hline Inclusion & Exclusion \\
\hline Bovine TB & Wrong disease \\
Cattle or buffalo & Wrong species \\
Used BCG to vaccinate (and & Wrong vaccine \\
specified the strain used) & \\
Evaluated efficacy of BCG vaccination & Wrong type of study \\
English & Language limitation: not in English \\
Full text of publication obtanied & Full-text unavailable \\
& Other
\end{tabular}

terms common to known articles of interest were evaluated before the following search terms were finalized: (BCG AND (("mycobacterium bovis" OR tuberculosis) AND (cows OR cattle OR bovine) AND (protect* OR effica* OR lesion* OR immune* OR vaccin*))). In order to minimize publication bias, search terms were kept uniform across the four databases (PubMed, $\mathrm{CAB}$, Biological Abstracts, and Web of Science), which were selected for their inclusion of major and minor international journals. No limits were placed on publication date, and only published studies were considered. EndNote X8, a citation software program, was used to organize the articles generated by the four databases as well as remove duplicate publications. The study conforms to Preferred Reporting Items for Systematic Reviews and Meta-analyses (PRISMA) guidelines (91).

\section{Study Inclusion Criteria}

Inclusion and exclusion criteria are detailed in Table 4. All included studies compared the potentially protective effects induced by a BCG variant against natural or experimental challenge with bTB in a vaccinated group of cattle and a control group of unvaccinated cattle. Studies with no control group, such as surveys evaluating the effectiveness of vaccination campaigns, were excluded. Additionally, articles that failed to report the BCG vaccine strain tested were excluded (summarized in Supplementary Table 1). While efforts were made to identify articles manually, all final included articles were represented in the formal database search. Primary studies were included when available. However, review articles were included when the primary article was inaccessible and adequate information on BCG strain, bTB challenge, and protection was detailed for vaccinate and control groups.

\section{Data Extraction}

A uniform data extraction form was developed based on information of interest from pre-identified articles and used by each of the extractors. Study identifiers included author, publication date, title, journal, study location, and study time period. Headings for study design included control and vaccinate group sizes; BCG strain (Danish 1331, Danish freeze-dried, Glaxo, Pasteur 1173P2, Tokyo); BCG dose; and BCG administration route (subcutaneous, endobronchial, intratracheal, intramuscular, oral); cattle age at time of vaccination; revaccination timing, frequency, and dose (if applicable); animal breed; and time from vaccination to 
TABLE 5 | A summary of all studies included in the meta-analysis.

\begin{tabular}{lllr}
\hline & & \multicolumn{2}{c}{ Infection } \\
\cline { 3 - 4 } & & Yes & No \\
\hline Vaccination & Yes & 362 & 1,540 \\
& No & 530 & 1,137 \\
\hline
\end{tabular}

Out of a total of 1,902 BCG vaccinated animals, 356 were found to be positive (either by culture and/or presence of visible lesions) for bTB while 1,502 remained negative. Similarly, out of 1,667 control animals, 520 were infected while 1,104 animals neither had lesions nor any growth in culture.

challenge. Headings detailing bTB challenge methods included challenge type (natural or experimental) and duration (from challenge to slaughter or end of study period); as well as challenge strain, route (intratracheal, subcutaneous, oral, endobronchial) and dose if challenge type was experimental. Headings for protection included the infection measurement method (postmortem, culture), number of vaccinates and controls affected, and the protection percentage reported. Vaccine efficacy was measured based on culture growth, and in the absence of culture data, presence or absence of visible lung or lymph node lesions in the control and treatment groups was used.

Prior to the formal review of all articles in the pool, data extractors (SS, LE, and $\mathrm{CH}$ ) conducted a pilot run of 20 random articles in order to test inclusion/exclusion criteria and finalize the data extraction form. SS, LE, and $\mathrm{CH}$ independently performed the formal review of all articles, which comprised an initial screening of article titles and abstracts for inclusion or exclusion. Full-text review for data extraction from included studies was performed by SS and LE. When discrepancies arose in study inclusion/exclusion or extracted data between reviewers, studies were collectively revisited and discussed in order to reach a final decision.

Included studies used common protection measurement methods, primarily post-mortem examination (PM) and/or culture. In the case that a study used both PM and culture to measure protection, preference was given to culture (65); in the case that lung and lymph node lesions were reported, we considered positivity per lung lesions for effect measure. We only included studies that specified the challenge strain. A majority of the included publications performed experimental (17/24) challenge and did not revaccinate animals (18/24). A complete list of all included and excluded studies is publicly available at https://doi.org/10.26208/pykx-8s25.

\section{Statistical Analysis}

All statistical analyses were performed in $\mathrm{R}$ (version 3.5.0) using RStudio (version 1.1.442). Full source codes used for the statistical analyses are publicly available with this publication at https://doi.org/10.26208/pykx-8s25. Random effects (RE) (4) and fixed effects (FE) meta-analysis models were estimated using the "rma" function of the "metaphor" package and analyzed using additional functions from the "meta" package (92, 93). Funnel plots were constructed to assess for systematic bias in the fitted models. Cochran's Q statistic was computed to test for unexplained heterogeneity, and Higgin's statistic helped describe the inconsistencies in studies' results $(94,95)$. Key parameters used to define protocols for BCG vaccination trials that were judged on biological grounds to be important were assessed as moderators in a multivariate meta-regression model, estimated using the rma function of the metafor package.

Due to the variability between reporting of pathology between different studies, vaccine efficacy was measured as a binary dichotomous variable based on the presence or absence of culture growth, or visible lesions in the control and treatment groups. Vaccine efficacy is most commonly measured using the relative risk or risk ratio (RR) (4), defined as the ratio of attack rate in the vaccinated and control groups. As well as being more easily interpretable than other measures (such as odds ratios and risk differences), RR can be used to define vaccine efficacy (1-RR) and was estimated here per the RE model. The $2 \times 2$ contingency table below provides a summary of included sample size (Table 5).

\section{Scenario Analysis}

Thus far, no study on BCG efficacy has attempted to estimate the efficacy of BCG to reduce the infectiousness of vaccinates who subsequently become infected. In the absence of empirical data, the reduction in lesions in vaccinated animals estimated from natural transmission studies may be considered as a proxy for reduction in infectiousness. While how the extent of lesions directly relates to a reduction in infectiousness and thus transmission risk was not quantified, this significant reduction in lesion severity following BCG vaccination likely contributes to reduction in risk of transmission from vaccinates to susceptible cattle. Here, we conducted scenario analyses to explore the potential implications for disease control and the importance of estimating this neglected-but critical-aspect of BCG efficacy. We consider this proportionate reduction in lesions as a plausible upper bound for the total vaccine-induced protection.

For a well-mixed population, the critical vaccination threshold $\left(V_{c}\right)$ for elimination of a disease can be calculated from the basic reproduction number $\left(\mathrm{R}_{0}\right)$ using the formula $\mathrm{V}_{\mathrm{c}}=(1-$ $\left.1 / R_{0}\right) / E$, where $E$ is the relative (combined direct and indirect) efficacy of the vaccine (96). For domesticated livestock species in organized settings, routine vaccinations are often performed for all animals, hence we can reframe the critical threshold in terms of the critical efficacy $\mathrm{E}_{\mathrm{c}}=\left(1-1 / R_{0}\right)$. Thus, with a direct vaccine efficacy of $25 \%$ (95\% CI: 18,32$)$, BCG could be expected to successfully eliminate infection from fully vaccinated herds when the within-herd $\mathrm{R}_{0}$ is 1.32 (95\% CI, 1.20-1.45) or lower.

A simple deterministic model for the within-herd transmission of bTB was used to explore the potential impact of BCG vaccination in an expanding dairy sector. The model assumes a well-mixed herd with animals stratified into four epidemiological compartments. In an unvaccinated population, animals are either susceptible $(S)$ or infected and potentially infectious $(I)$. Vaccinated individuals $(V)$ have a reduced risk of becoming infected and we add a final infected after vaccination compartment to model a reduced infectiousness for these animals $\left(I_{V}\right)$. Density dependence in transmission is modeled using the non-linear relationship estimated from herds in Great Britain (GB) using the so-called SOR model (71). The SOR model 
subdivides the infectious class into an occult $(\mathrm{O})$ and reactive (R) groups that differ in their reaction to the tuberculin skin test, but also assumes infected animals are potentially infectious immediately and is thus structurally equivalent to the $\operatorname{SI}\left(V I_{V}\right)$ model used here.

The full model equations are:

$$
\begin{aligned}
\frac{d S}{d t} & =(1-p) \mu N-\frac{\beta}{\left(N / N_{m}\right)^{q}}\left(I+\left(1-\varepsilon_{I}\right) I v\right) S-v S \\
\frac{d I}{d t} & =\frac{\beta}{\left(N / N_{m}\right)^{q}}\left(I+\left(1-\varepsilon_{I}\right) I_{V}\right) S-v I \\
\frac{d V}{d t} & =p \mu N-\left(1-\varepsilon_{S}\right) \frac{\beta}{\left(N / N_{m}\right)^{q}}\left(I+\left(1-\varepsilon_{I}\right) I_{V}\right) V-v V \\
\frac{d I_{V}}{d t} & =\left(1-\varepsilon_{s}\right) \frac{\beta}{\left(N / N_{m}\right)^{q}}\left(I+\left(1-\varepsilon_{I}\right) I_{V}\right) V-v I_{V}
\end{aligned}
$$$$
\frac{d C}{d t}=\frac{\beta}{\left(N / N_{m}\right)^{q}}\left(I+\left(1-\varepsilon_{I}\right) I_{V}\right) S+\left(1-\varepsilon_{s}\right)
$$$$
\frac{\beta}{\left(N / N_{m}\right)^{q}}\left(I+\left(1-\varepsilon_{I}\right) I_{V}\right) V
$$$$
\frac{d N}{d t}=(\mu-v) N
$$

where $\varepsilon_{s}$ is the efficacy of vaccination to protect against infection (reduction in susceptibility), $\varepsilon_{I}$ is the efficacy of vaccination with respect to reducing the infectiousness of vaccinates that subsequently become infected and $N_{m}$ is a constant centering parameter $(=165)$ used for the estimation of $\mathrm{q}$ in the original GB model. $\beta$ is a transmission parameter which we fix using the assumed initial prevalence $\left(\mathrm{I}_{0}\right)$ and initial herd size $\left(\mathrm{N}_{0}\right)$ for each scenario and $\mathrm{q}$ measures the strength of density dependence of transmission with $\mathrm{q}=0$ corresponding to density dependence and $\mathrm{q}=1$ frequency dependence. For the default scenario we use the point estimate from Conlan et al. (71) of $\mathrm{q}=0.15$ and present an alternative weaker density dependent scenario ( $q=0.71$, upper bound of approximate posterior distribution) in Supplementary Material.

For illustration we consider a herd with initial size $\mathrm{N}_{0}=30$, a $20 \%$ per annum replacement rate $(v=0.2$ per year $)$ and per

\section{REFERENCES}

1. Cousins DV. Mycobacterium bovis infection and control in domestic livestock. Rev Sci Tech. (2001) 20:71-85. doi: 10.20506/rst.20.1.1263

2. Doyle TM, Stuart P. Vaccination of Cattle with B.C.G. Br Vet J. (1958) 114:3-10. doi: 10.1016/S0007-1935(17)45652-1

3. Haring CM, Traum J, Hayes FM, Henry BS. Vaccination of calves against tuberculosis with Calmette-Guérin Culture, BCG. Hilgardia J Agric Sci. (1930) 4:307-94. doi: 10.3733/hilg.v04n12p307

4. Vordermeier HM, Jones GJ, Buddle BM, Hewinson RG, VillarrealRamos B. Bovine tuberculosis in cattle: vaccines, DIVA tests, and host biomarker discovery. Annu Rev Anim Biosci. (2016) 4:87-109. doi: 10.1146/annurev-animal-021815-111311 capita birth rate $\mu=1.15 v$ to give a population growth rate of $15 \%$ per year $\left(N=N_{0} e^{-(\mu-v) t}\right)$.

The basic reproduction ratio for this model (for fixed herd size $\mathrm{N}_{0}$ ) can be calculated (next generation method) as:

$$
R_{0}=\frac{\beta}{v} N_{0}\left(N_{0} / N_{m}\right)^{-q}
$$

We set the (97) value of $\beta$ for each scenario by assuming that the initial population is at the equilibrium point of the constant population model. Thus, at $t=0$, we assume that:

$$
R_{0}=1 /\left(1-I_{0} / N_{0}\right)
$$

and thus set:

$$
\beta=\frac{\nu R_{0}\left(N_{0} / N_{m}\right)^{q}}{N_{0}}
$$

Full source codes used for the statistical analyses are publicly available with this publication at https://doi.org/10.26208/pykx8 s25.

\section{AUTHOR CONTRIBUTIONS}

SS, LE, and VK designed the study. SS, LE, and $\mathrm{CH}$ performed data extraction. SS and AC compiled the final data and analyzed the data. SS drafted the paper. SS, AC, PD, MVe, GA, GR, JW, NJ, DB, MVo, and VK contributed to the writing. All authors contributed to the article and approved the submitted version.

\section{FUNDING}

This study was supported by a grant (OPP1176950) from the Bill \& Melinda Gates Foundation and the U.K. Department for International Development.

\section{SUPPLEMENTARY MATERIAL}

The Supplementary Material for this article can be found online at: https://www.frontiersin.org/articles/10.3389/fvets. 2021.637580/full\#supplementary-material 
8. Srinivasan S, Jones G, Veerasami M, Steinbach S, Holder T, Zewude A, et al. A defined antigen skin test for the diagnosis of bovine tuberculosis. Sci Adv. (2019) 5:eaax4899. doi: 10.1126/sciadv.aax4899

9. Srinivasan S, Subramanian S, Shankar Balakrishnan S, Ramaiyan Selvaraju K, Manomohan V, Selladurai S, et al. A defined antigen skin test that enables implementation of BCG vaccination for control of bovine tuberculosis: proof of concept. Front Vet Sci. (2020) 7:391-391. doi: 10.3389/fvets.2020.00391

10. Buddle BM, Vordermeier HM, Chambers MA, De Klerk-Lorist LM. Efficacy and safety of BCG vaccine for control of tuberculosis in domestic livestock and wildlife. Front Vet Sci. (2018) 5:259-259. doi: 10.3389/fvets.2018.00259

11. Conlan AJK, Vordermeier M, De Jong MCM, Wood JLN. The intractable challenge of evaluating cattle vaccination as a control for bovine tuberculosis. eLife. (2018) 7:e27694. doi: 10.7554/eLife.27694

12. Waddington FG, Ellwood DC. An experiment to challenge the resistance to tuberculosis in B.C.G. vaccinated cattle in Malawi. Br Vet J. (1972) 128:541-52. doi: 10.1016/S0007-1935(17)36683-6

13. Berggren SA. Field experiment with BCG vaccine in Malawi. Br Vet J. (1981) 137:88-96. doi: 10.1016/S0007-1935(17)31792-X

14. Buddle BM, De Lisle GW, Pfeffer A, Aldwell FE. Immunological responses and protection against Mycobacterium bovis in calves vaccinated with a low dose of BCG. Vaccine. (1995) 13:1123-30. doi: 10.1016/0264-410X(94)00055-R

15. Buddle BM, Keen D, Thomson A, Jowett G, Mccarthy AR, Heslop J, et al. Protection of cattle from bovine tuberculosis by vaccination with BCG by the respiratory or subcutaneous route, but not by vaccination with killed Mycobacterium vaccae. Res Vet Sci. (1995) 59:10-6. doi: 10.1016/0034-5288(95)90023-3

16. Buddle BM, Parlane NA, Keen DL, Aldwell FE, Pollock JM, Lightbody K, et al. Differentiation between Mycobacterium bovis BCG-vaccinated and M. bovisinfected cattle by using recombinant mycobacterial antigens. Clin Diagn Lab Immunol. (1999) 6:1-5. doi: 10.1128/CDLI.6.1.1-5.1999

17. Buddle BM, Wards BJ, Aldwell FE, Collins DM, De Lisle GW. Influence of sensitisation to environmental mycobacteria on subsequent vaccination against bovine tuberculosis. Vaccine. (2002) 20:1126-33. doi: 10.1016/S0264-410X(01)00436-4

18. Buddle BM, Wedlock DN, Parlane NA, Corner LA, De Lisle GW, Skinner MA. Revaccination of neonatal calves with Mycobacterium bovis BCG reduces the level of protection against bovine tuberculosis induced by a single vaccination. Infect Immun. (2003) 71:6411-9. doi: 10.1128/IAI.71.11.6411-6419.2003

19. Buddle BM, Aldwell FE, Skinner MA, De Lisle GW, Denis M, Vordermeier $\mathrm{HM}$, et al. Effect of oral vaccination of cattle with lipid-formulated BCG on immune responses and protection against bovine tuberculosis. Vaccine. (2005) 23:3581-9. doi: 10.1016/j.vaccine.2005.01.150

20. Wedlock DN, Denis M, Vordermeier HM, Hewinson RG, Buddle BM. Vaccination of cattle with Danish and Pasteur strains of Mycobacterium bovis BCG induce different levels of IFNgamma post-vaccination, but induce similar levels of protection against bovine tuberculosis. Vet Immunol Immunopathol. (2007) 118:50-8. doi: 10.1016/j.vetimm.2007.04.005

21. Buddle BM, Denis M, Aldwell FE, Martin Vordermeier H, Glyn Hewinson $\mathrm{R}$, Neil Wedlock D. Vaccination of cattle with Mycobacterium bovis BCG by a combination of systemic and oral routes. Tuberculosis (Edinb). (2008) 88:595-600. doi: 10.1016/j.tube.2008.01.005

22. Wedlock DN, Denis M, Painter GF, Ainge GD, Vordermeier HM, Hewinson RG, et al. Enhanced protection against bovine tuberculosis after coadministration of Mycobacterium bovis BCG with a Mycobacterial protein vaccine-adjuvant combination but not after coadministration of adjuvant alone. Clin Vaccine Immunol. (2008) 15:765-72. doi: 10.1128/CVI.00034-08

23. Ameni G, Vordermeier M, Aseffa A, Young DB, Hewinson RG. Field evaluation of the efficacy of Mycobacterium bovis bacillus Calmette-Guerin against bovine tuberculosis in neonatal calves in Ethiopia. Clin Vaccine Immunol. (2010) 17:1533-8. doi: 10.1128/CVI.00222-10

24. De Klerk LM, Michel AL, Bengis RG, Kriek NP, Godfroid J. BCG vaccination failed to protect yearling African buffaloes (Syncerus caffer) against experimental intratonsilar challenge with Mycobacterium bovis. Vet Immunol Immunopathol. (2010) 137:84-92. doi: 10.1016/j.vetimm.2010.04.013

25. Lopez-Valencia G, Renteria-Evangelista T, Williams JEJ, Licea-Navarro A, Mora-Valle AEL, Medina-Basulto G. Field evaluation of the protective efficacy of Mycobacterium bovis BCG vaccine against bovine tuberculosis. Res Vet Sci. (2010) 88:44-9. doi: 10.1016/j.rvsc.2009.05.022
26. Buddle BM, Aldwell FE, De Lisle GW, Vordermeier HM, Hewinson RG, Wedlock DN. Low oral BCG doses fail to protect cattle against an experimental challenge with Mycobacterium bovis. Tuberculosis (Edinb). (2011) 91:400-5. doi: 10.1016/j.tube.2011.07.001

27. Hope JC, Thom ML, Mcaulay M, Mead E, Vordermeier HM, Clifford D, et al. Identification of surrogates and correlates of protection in protective immunity against Mycobacterium bovis infection induced in neonatal calves by vaccination with $M$. bovis BCG Pasteur and M. bovis BCG Danish Clin Vaccine Immunol. (2011) 18:373-9. doi: 10.1128/CVI.00543-10

28. Thom ML, Mcaulay M, Vordermeier HM, Clifford D, Hewinson RG, Villarreal-Ramos B, et al. Duration of immunity against Mycobacterium bovis following neonatal vaccination with bacillus Calmette-Guérin Danish: significant protection against infection at 12 , but not 24, months. Clin Vaccine Immunol. (2012) 19:1254-60. doi: 10.1128/CVI.00301-12

29. Buddle BM, Hewinson RG, Vordermeier HM, Wedlock DN. Subcutaneous administration of a 10-fold-lower dose of a commercial human tuberculosis vaccine, Mycobacterium bovis bacillus Calmette-Guerin Danish, induced levels of protection against bovine tuberculosis and responses in the tuberculin intradermal test similar to those induced by a standard cattle dose. Clin Vaccine Immunol. (2013) 20:1559-62. doi: 10.1128/CVI.00435-13

30. Dean G, Whelan A, Clifford D, Salguero FJ, Xing Z, Gilbert S, et al. Comparison of the immunogenicity and protection against bovine tuberculosis following immunization by BCG-priming and boosting with adenovirus or protein based vaccines. Vaccine. (2014) 32:1304-10. doi: 10.1016/j.vaccine.2013.11.045

31. Parlane NA, Shu D, Subharat S, Wedlock DN, Rehm BH, De Lisle GW, et al. Revaccination of cattle with bacille Calmette-Guérin two years after first vaccination when immunity has waned, boosted protection against challenge with Mycobacterium bovis. PLoS ONE. (2014) 9:e106519. doi: 10.1371/journal.pone.0106519

32. Dean GS, Clifford D, Whelan AO, Tchilian EZ, Beverley PC, Salguero FJ, et al. Protection induced by simultaneous subcutaneous and endobronchial vaccination with BCG/BCG and BCG/adenovirus expressing antigen 85A against Mycobacterium bovis in cattle. PLoS ONE. (2015) 10:e0142270. doi: 10.1371/journal.pone.0142270

33. Buddle BM, Shu D, Parlane NA, Subharat S, Heiser A, Hewinson RG, et al. Vaccination of cattle with a high dose of BCG vaccine 3 weeks after experimental infection with Mycobacterium bovis increased the inflammatory response, but not tuberculous pathology. Tuberculosis (Edinb). (2016) 99:1207. doi: 10.1016/j.tube.2016.05.004

34. Nugent G, Yockney IJ, Whitford J, Aldwell FE, Buddle BM. Efficacy of oral BCG vaccination in protecting free-ranging cattle from natural infection by Mycobacterium bovis. Vet Microbiol. (2017) 208:181-9. doi: 10.1016/j.vetmic.2017.07.029

35. Ameni G, Tafess K, Zewde A, Eguale T, Tilahun M, Hailu T, et al. Vaccination of calves with Mycobacterium bovis Bacillus Calmette-Guerin reduces the frequency and severity of lesions of bovine tuberculosis under a natural transmission setting in Ethiopia. Transbound Emerg Dis. (2018) 65:96-104. doi: $10.1111 /$ tbed.12618

36. Nugent G, Yockney IJ, Cross ML, Buddle BM. Low-dose BCG vaccination protects free-ranging cattle against naturally-acquired bovine tuberculosis. Vaccine. (2018) 36:7338-44. doi: 10.1016/j.vaccine.2018.10.025

37. Viechtbauer W. Accounting for heterogeneity via random-effects models and moderator analyses in meta-analysis. J Psychol. (2007) 215:104-21. doi: 10.1027/0044-3409.215.2.104

38. Mavridis D, Salanti G. How to assess publication bias: funnel plot, trim-andfill method and selection models. Evid Based Mental Health. (2014) 17:30. doi: 10.1136/eb-2013-101699

39. Duval S, Tweedie R. Trim and fill: a simple funnel-plot-based method of testing and adjusting for publication bias in meta-analysis. Biometrics. (2000) 56:455-63. doi: 10.1111/j.0006-341X.2000.00455.x

40. Remlinger P, Bailly J. Nasal immunization against tuberculosis. Compt Rend Soc Biol. (1928) 99:1556-7.

41. Conlan AJK, Wood JLN. The Epidemiology of Mycobacterium bovis Infection in Cattle, Chapter 4, Bovine Tuberculosis (2018). Available online at: https:// www.cabi.org/vetmedresource/ebook/20183117961

42. Srinivasan S, Easterling L, Rimal B, Niu XM, Conlan AJK, Dudas P, et al. Prevalence of bovine tuberculosis in India: a systematic review and 
meta-analysis. Transbound Emerg Dis. (2018) 65:1627-40. doi: 10.1111/tbed. 12915

43. Halloran ME, Struchiner CJ, Longini IM Jr. Study designs for evaluating different efficacy and effectiveness aspects of vaccines. Am J Epidemiol. (1997) 146:789-803.

44. Watson E. Studies on bacillus Calmette-Guérin (BCG) and vaccination against tuberculosis. Can J Res. (1933) 9:128-36. doi: 10.1139/cjr33-073

45. WHO/FAO Expert Committee on Zoonoses. Second Report. World Health Organisation Technical Report Series No 169. (1959)

46. Various papers on Calmette's BCG. Ann Inst Pasteur. (1927) 41:201-368.

47. Gerlach F. Preventive inoculation against tuberculosis with Calmette's BCG. $Z$ Immun U Exp Therap. (1927) 51:256-75.

48. Watson EA. Report of the Pathological Division, Health of Animals Branch, Department of Agriculture, for the year ending March 81st, 1930. Ottawa, ON: F. A. Acland (1930).

49. Calmette A. Preventive vaccination against tuberculosis with BCG. Proc R Soc Med. (1931) 24:1481-90. doi: 10.1177/003591573102401109

50. Gerlach F. Vaccination Against Tuberculosis (1931). Available online at: https://www.cabdirect.org/cabdirect/abstract/19322200716

51. Gloyne SR. BCG Vaccine-A Review of Its Uses (1931).

52. Griffith AS, Buxton JB, Glover RE. Further Results of Immunity Experiments on Calves With the BCG Vaccine. Cambridge: University of Cambridge (1931).

53. Gerlach F. Results of BCG vaccination on cattle in Austria. Wien Tierarztl Monatssch. (1932) 19:353-66.

54. Young JA, Paterson JS. Studies on the vaccination of cattle as a measure against infection with tuberculosis with the living vole acid-fast bacillus. J Hyg. (London). (1949) 4:39-78.

55. Young JA. B.C.G. vaccination. Lancet. (1949) 254:1055-6. doi: 10.1016/S0140-6736(49)91629-3

56. Ellwood DC, Waddington FG. A second experiment to challenge the resistance to tuberculosis in B.C.G. vaccinated cattle in Malawi. Br Vet J. (1972) 128:619-26. doi: 10.1016/S0007-1935(17)36634-4

57. Ellwood DC. First results of the field use of BCG vaccine to control bovine tuberculosis in Malawi. Br Vet J. (1975) 131:186-9. doi: 10.1016/S0007-1935(17)35339-3

58. Waters WR, Palmer MV, Buddle BM, Vordermeier HM. Bovine tuberculosis vaccine research: historical perspectives and recent advances. Vaccine. (2012) 30:2611-22. doi: 10.1016/j.vaccine.2012.02.018

59. Olea-Popelka F, Muwonge A, Perera A, Dean AS, Mumford E, ErlacherVindel E, et al. Zoonotic tuberculosis in human beings caused by Mycobacterium bovis-a call for action. Lancet Infect Dis. (2017) 17:e21-5. doi: 10.1016/S1473-3099(16)30139-6

60. Hope JC, Thom ML, Villarreal-Ramos B, Vordermeier HM, Hewinson RG, Howard CJ. Vaccination of neonatal calves with Mycobacterium bovis BCG induces protection against intranasal challenge with virulent $M$. bovis. Clin Exp Immunol. (2005) 139:48-56. doi: 10.1111/j.1365-2249.2005. 02668.x

61. Gopinath K, Singh S. Non-tuberculous mycobacteria in TB-endemic countries: are we neglecting the danger? PLoS Negl Trop Dis. (2010) 4:e615. doi: 10.1371/journal.pntd.0000615

62. Hope JC, Thom ML, Villarreal-Ramos B, Vordermeier HM, Hewinson RG, Howard CJ. Exposure to Mycobacterium avium induces lowlevel protection from Mycobacterium bovis infection but compromises diagnosis of disease in cattle. Clin Exp Immunol. (2005) 141:432-9. doi: 10.1111/j.1365-2249.2005.02882.x

63. Nuru A, Zewude A, Mohammed T, Wondale B, Teshome L, Getahun M, et al. Nontuberculosis mycobacteria are the major causes of tuberculosis like lesions in cattle slaughtered at Bahir Dar Abattoir, northwestern Ethiopia. BMC Vet Res. (2017) 13:237. doi: 10.1186/s12917-017-1168-3

64. Sweetline Anne N, Ronald BSM, Kumar TMS, Kannan P, Thangavelu A. Molecular identification of Mycobacterium tuberculosis in cattle. Vet Microbiol. (2017) 198:81-7. doi: 10.1016/j.vetmic.2016.12.013

65. De La Rua-Domenech R, Goodchild AT, Vordermeier HM, Hewinson RG, Christiansen $\mathrm{KH}$, Clifton-Hadley RS. Ante mortem diagnosis of tuberculosis in cattle: a review of the tuberculin tests, gamma-interferon assay and other ancillary diagnostic techniques. Res Vet Sci. (2006) 81:190-210. doi: 10.1016/j.rvsc.2005.11.005
66. Shim E, Galvani AP. Distinguishing vaccine efficacy and effectiveness. Vaccine. (2012) 30:6700-5. doi: 10.1016/j.vaccine.2012.08.045

67. Halloran ME, Haber M, Longini IM Jr., Struchiner CJ. Direct and indirect effects in vaccine efficacy and effectiveness. Am J Epidemiol. (1991) 133:32331. doi: 10.1093/oxfordjournals.aje.a115884

68. Carter SP, Chambers MA, Rushton SP, Shirley MDF, Schuchert P, Pietravalle $\mathrm{S}$, et al. BCG vaccination reduces risk of tuberculosis infection in vaccinated badgers and unvaccinated Badger Cubs. PLoS ONE. (2012) 7:e49833. doi: 10.1371/journal.pone.0049833

69. Skuce RA, Allen AR, Mcdowell SW. Herd-level risk factors for bovine tuberculosis: a literature review. Vet Med Int. (2012) 2012:621210. doi: $10.1155 / 2012 / 621210$

70. Barlow ND, Kean JM, Hickling G, Livingstone PG, Robson AB. A simulation model for the spread of bovine tuberculosis within New Zealand cattle herds. Prev Vet Med. (1997) 32:57-75. doi: 10.1016/S0167-5877(97)00002-0

71. Conlan AJK, Mckinley TJ, Karolemeas K, Pollock EB, Goodchild AV, Mitchell AP, et al. Estimating the hidden burden of bovine tuberculosis in Great Britain. PLoS Comput Biol. (2012) 8:e1002730. doi: 10.1371/journal.pcbi.1002730

72. Mekonnen GA, Conlan AJK, Berg S, Ayele BT, Alemu A, Guta S, et al. Prevalence of bovine tuberculosis and its associated risk factors in the emerging dairy belts of regional cities in Ethiopia. Prev Vet Med. (2019) 168:81-9. doi: 10.1016/j.prevetmed.2019.04.010

73. Balseiro A, Prieto JM, Álvarez V, Lesellier S, Davé D, Salguero FJ, et al. Protective effect of oral BCG and inactivated Mycobacterium bovis vaccines in European Badgers (Meles meles) experimentally infected with M. bovis. Front Vet Sci. (2020) 7:41. doi: 10.3389/fvets.2020.00041

74. Ameni G, Aseffa A, Sirak A, Engers H, Young DB, Hewinson RG, et al. Effect of skin testing and segregation on the prevalence of bovine tuberculosis, and molecular typing of Mycobacterium bovis, in Ethiopia. Vet Rec. (2007) 161:782-6.

75. Dean GS, Rhodes SG, Coad M, Whelan AO, Wheeler P, VillarealRamos B, et al. Isoniazid treatment of Mycobacterium bovis in cattle as a model for human tuberculosis. Tuberculosis. (2008) 88:586-94. doi: 10.1016/j.tube.2008.01.004

76. Thoen CO, Steele JH, Gilsdorf MJ. Mycobacterium bovis Infection in Animals and Humans. Ames, IA: Blackwell Publishing (2008). p. 329.

77. McLaughlin AM, Gibbons N, Fitzgibbon M, Power JT, Foley SC, Hayes JP, et al. Primary isoniazid resistance in Mycobacterium bovis disease: a prospect of concern. Am J Respir Crit Care Med. (2012) 186:110-1. doi: 10.1164/ajrccm.186.1.110

78. Skinner MA, Ramsay AJ, Buchan GS, Keen DL, Ranasinghe C, Slobbe $\mathrm{L}$, et al. A DNA prime-live vaccine boost strategy in mice can augment IFN- $\gamma$ responses to mycobacterial antigens but does not increase the protective efficacy of two attenuated strains of Mycobacterium bovis against bovine tuberculosis. Immunology. (2003) 108:548-55. doi: 10.1046/j.1365-2567.2003.01589.x

79. Vordermeier HM, Rhodes SG, Dean G, Goonetilleke N, Huygen K, Hill AVS, et al. Cellular immune responses induced in cattle by heterologous primeboost vaccination using recombinant viruses and bacille Calmette-Guérin. Immunology. (2004) 112:461-70. doi: 10.1111/j.1365-2567.2004.01903.x

80. Skinner MA, Wedlock DN, De Lisle GW, Cooke MM, Tascon RE, Ferraz JC, et al. The order of prime-boost vaccination of neonatal calves with Mycobacterium bovis BCG and a DNA vaccine encoding mycobacterial proteins Hsp65, Hsp70, and Apa is not critical for enhancing protection against bovine tuberculosis. Infect Immun. (2005) 73:4441-4. doi: 10.1128/IAI.73.7.4441-4444.2005

81. Vordermeier HM, Huygen K, Singh M, Hewinson RG, Xing Z. Immune responses induced in cattle by vaccination with a recombinant adenovirus expressing mycobacterial antigen 85A and Mycobacterium bovis BCG. Infect Immun. (2006) 74:1416-8. doi: 10.1128/IAI.74.2.1416-1418.2006

82. Maue AC, Waters WR, Palmer MV, Nonnecke BJ, Minion EC, Browne WC, et al. An ESAT-6 : CFP10 DNA vaccine administered in conjunction with Mycobacterium bovis BCG confers protection to cattle challenged with virulent M. bovis. Vaccine. (2007) 25:4735-46. doi: 10.1016/j.vaccine.2007.03.052

83. Garrido JM, Sevilla IA, Beltrán-Beck B, Minguijón E, Ballesteros C, Galindo $\mathrm{RC}$, et al. Protection against tuberculosis in Eurasian wild boar vaccinated 
with heat-inactivated Mycobacterium bovis. PLoS ONE. (2011) 6:e24905. doi: 10.1371/journal.pone.0024905

84. Beltrán-Beck B, Romero B, Boadella M, Casal C, Bezos J, Mazariegos M, et al. Tonsils of the soft palate do not mediate the response of pigs to oral vaccination with heat-inactivated Mycobacterium bovis. Clin Vaccine Immunol. (2014) 21:1128-36. doi: 10.1128/CVI.00221-14

85. Thomas J, Risalde M, Serrano M, Sevilla I, Geijo M, Ortíz JA, et al. The response of red deer to oral administration of heat-inactivated Mycobacterium bovis and challenge with a field strain. Vet Microbiol. (2017) 208:195-202. doi: 10.1016/j.vetmic.2017.08.007

86. Díez-Delgado I, Sevilla IA, Romero B, Tanner E, Barasona JA, White AR, et al. Impact of piglet oral vaccination against tuberculosis in endemic free-ranging wild boar populations. Prev Vet Med. (2018) 155:11-20. doi: 10.1016/j.prevetmed.2018.04.002

87. Risalde MA, López V, Contreras M, Mateos-Hernández L, Gortázar C, De La Fuente J. Control of mycobacteriosis in zebrafish (Danio rerio) mucosally vaccinated with heat-inactivated Mycobacterium bovis. Vaccine. (2018) 36:4447-53. doi: 10.1016/j.vaccine.2018.06.042

88. Roy Á, Risalde MA, Bezos J, Casal C, Romero B, Sevilla I, et al. Response of goats to intramuscular vaccination with heat-killed Mycobacterium bovis and natural challenge. Comp Immunol Microbiol Infect Dis. (2018) 60:28-34. doi: 10.1016/j.cimid.2018.09.006

89. Serrano M, Elguezabal N, Sevilla IA, Geijo MV, Molina E, Arrazuria $\mathrm{R}$, et al. Tuberculosis detection in paratuberculosis vaccinated calves: new alternatives against interference. PLoS ONE. (2017) 12:e0169735. doi: 10.1371/journal.pone.0169735

90. Serrano M, Elguezabal N, Sevilla IA, Geijo MV, Molina E, Juste RA, et al. Preliminary results indicate that inactivated vaccine against paratuberculosis could modify the course of experimental Mycobacterium bovis infection in calves. Front Vet Sci. (2017) 4:175-175. doi: 10.3389/fvets.2017.00175

91. Liberati A, Altman DG, Tetzlaff J, Mulrow C, Gotzsche PC, Ioannidis JP, et al. The PRISMA statement for reporting systematic reviews and meta-analyses of studies that evaluate health care interventions: explanation and elaboration. PLoS Med. (2009) 6:e1000100. doi: 10.1371/journal.pmed.1000100

92. Schwarzer G. meta: An R package for meta-analysis. R News. (2007) 7:40-5.

93. Viechtbauer $\mathrm{W}$. Conducting meta-analyses in $\mathrm{R}$ with the metafor package. $J$ Stat Softw. (2010) 36:48. doi: 10.18637/jss.v036.i03

94. Cochran WG. The combination of estimates from different experiments. Biometrics. (1954) 10:101-29. doi: 10.2307/3001666

95. Higgins JP, Thompson SG, Deeks JJ, Altman DG. Measuring inconsistency in meta-analyses. BMJ. (2003) 327:557-60. doi: 10.1136/bmj.327.7414.557

96. Anderson RM. Infectious diseases of humans: dynamics and control. Epidemiol Infect. (1991) 108:211.

97. Pires D, Valente E, Simoes MF, Carmo N, Testa B, Constantino L, et al. Esters of pyrazinoic acid are active against pyrazinamide-resistant strains of Mycobacterium tuberculosis and other naturally resistant mycobacteria in vitro and ex vivo within macrophages. Antimicrob Agents Chemother. (2015) 59:7693-9. doi: 10.1128/AAC.00936-15

Conflict of Interest: MV is the Director of Cisgen Biotech Discoveries Pvt. Ltd., Chennai, India.

The remaining authors declare that the research was conducted in the absence of any commercial or financial relationships that could be construed as a potential conflict of interest.

Copyright (๑) 2021 Srinivasan, Conlan, Easterling, Herrera, Dandapat, Veerasami, Ameni, Jindal, Raj, Wood, Juleff, Bakker, Vordermeier and Kapur. This is an openaccess article distributed under the terms of the Creative Commons Attribution License (CC BY). The use, distribution or reproduction in other forums is permitted, provided the original author(s) and the copyright owner(s) are credited and that the original publication in this journal is cited, in accordance with accepted academic practice. No use, distribution or reproduction is permitted which does not comply with these terms. 